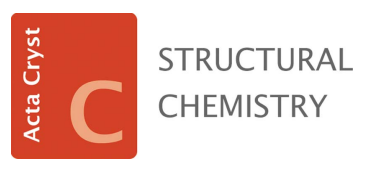

ISSN 2053-2296

Received 29 June 2020

Accepted 9 August 2020

Edited by M. Gardiner, Australian National University, Australia

Keywords: benzothiazinone; BTZ043; benzisothiazolinone; ring contraction; crystal structure; antimycobacterial activity.

CCDC references: 2022514; 2022515

Supporting information: this article has supporting information at journals.iucr.org/C

\section{Crystallographic evidence for unintended benzisothiazolinone 1-oxide formation from benzothiazinones through oxidation}

\author{
Tamira Eckhardt, ${ }^{a}$ Richard Goddard, ${ }^{\mathrm{b}}$ Christoph Lehmann, ${ }^{\mathrm{a}}$ Adrian Richter, ${ }^{\mathrm{a}}$ \\ Henok Asfaw Sahile, ${ }^{\text {c }}$ Rui Liu, ${ }^{\text {d }}$ Rohit Tiwari, ${ }^{\text {d }}$ Allen G. Oliver, ${ }^{\text {d Marvin J. Miller, }}$, \\ Rüdiger W. Seidel ${ }^{\mathrm{a} *}$ and Peter Imming $^{\mathrm{a}}$
}

\footnotetext{
anstitut für Pharmazie, Martin-Luther-Universität Halle-Wittenberg, Wolfgang-Langenbeck-Strasse 4, 06120 Halle (Saale), Germany, ${ }^{\mathbf{b}}$ Max-Planck-Institut für Kohlenforschung, Kaiser-Wilhelm-Platz 1, 45470 Mülheim an der Ruhr, Germany, ${ }^{\mathbf{c}}$ Department of Medicine and Department of Microbiology and Immunology, University of British Columbia, Vancouver, British Columbia, V6T 1Z3, Canada, and d Department of Chemistry and Biochemistry, University of Notre Dame, Indiana 46556, USA. *Correspondence e-mail: ruediger.seidel@pharmazie.uni-halle.de
}

1,3-Benzothiazin-4-ones (BTZs) are a promising new class of drugs with activity against Mycobacterium tuberculosis, which have already reached clinical trials. A product obtained in low yield upon treatment of 8-nitro-2-(piperidin-1-yl)-6(trifluoromethyl)-4H-benzothiazin-4-one with 3-chloroperbenzoic acid, in ana$\operatorname{logy}$ to a literature report describing the formation of sulfoxide and sulfone derived from BTZ043 [Tiwari et al. (2015). ACS Med. Chem. Lett. 6, 128-133], is a ring-contracted benzisothiazolinone (BIT) 1-oxide, namely, 7-nitro-2-(piperidine-1-carbonyl)-5-(trifluoromethyl)benzo[ $d]$ isothiazol-3(2H)-one 1-oxide, $\mathrm{C}_{14} \mathrm{H}_{12}$ $\mathrm{F}_{3} \mathrm{~N}_{3} \mathrm{O}_{5} \mathrm{~S}$, as revealed by X-ray crystallography. Single-crystal X-ray analysis of the oxidation product originally assigned as BTZ043 sulfone provides clear evidence that the structure of the purported BTZ043 sulfone is likewise the corresponding BIT 1-oxide, namely, 2-[(S)-2-methyl-1,4-dioxa-8-azaspiro[4.5]decane-8-carbonyl]-7-nitro-5-(trifluoromethyl)benzo[d]isothiazol-3(2H)-one 1-oxide, $\mathrm{C}_{17} \mathrm{H}_{16} \mathrm{~F}_{3} \mathrm{~N}_{3} \mathrm{O}_{7} \mathrm{~S}$. A possible mechanism for the ring contraction affording the BIT 1-oxides instead of the anticipated constitutionally isomeric BTZ sulfones and antimycobacterial activities thereof are discussed.

\section{Introduction}

Due to extremely low cidal concentrations against Mycobacterium tuberculosis in vitro, 8-nitro-1,3-benzothiazin-4ones (BTZs) have been the focus of many chemical, pharmacological and, recently, clinical studies (Mikušová et al., 2014; Kloss et al., 2017; Makarov \& Mikušová, 2020). Several promising compounds with improved aqueous solubilities have been identified with potent antitubercular activity (Zhang et al., 2019). The first small molecule crystal structure of a BTZ, namely macozinone (PBTZ169), was reported in this journal by Zhang \& Aldrich (2019). So far, the excellent in vitro activity appears not to translate to the low daily doses aspired for a medication that needs to be administered for months (Lupien et al., 2018). This could be attributed to pharmacokinetic problems and rapid metabolism by gut bacteria (Lv et al., 2017).

Research interest in this compound class is also inspired by the chemical versatility of the BTZs, which offer several points of attack, especially for nucleophiles and reducing agents (Tiwari et al., 2013). In turn, the BTZ S atom appears to be not very susceptible to oxidation. When BTZ043 (Scheme 1) was treated with the oxidizing agent 3-chloroperbenzoic acid at room temperature for several days, a major amount of 
unreacted BTZ starting material was recovered and small quantities of two oxidation products were isolated. Based on ${ }^{1} \mathrm{H}$ NMR spectroscopy and the sum formula calculated from high-resolution mass spectrometry, the corresponding BTZ sulfoxide and sulfone structures were assigned (Tiwari et al., 2015).<smiles></smiles><smiles>O=c1nc(N2CCCCC2)sc2c([N+](=O)[O-])cc(C(F)(F)F)cc12</smiles>

Treatment of 8-nitro-2-(piperidin-1-yl)-6-(trifluoromethyl)$4 H$-benzothiazin-4-one (1, Scheme 1) with 3-chloroperbenzoic acid in a similar way and crystallographic characterization of one of the oxidation products revealed the formation of a ringcontracted benzisothiazolone (BIT) 1-oxide instead of the anticipated BTZ sulfone (Fig. 1). Subsequent crystallographic reinvestigation of the BTZ043 oxidation product originally described as BTZ sulfone by us (Tiwari et al., 2015) evidenced that the structure must be revised to the corresponding ringcontracted BIT 1-oxide. In this article, we report the structural characterization of BIT 1-oxides resulting from oxidation of $\mathbf{1}$ and BTZ043, and propose a reaction mechanism of the ring contraction. We furthermore show by analysis of spectroscopic data and deliberate synthesis that the purported BTZ sulfoxide is actually a BIT.

\section{Experimental}

\subsection{General}

Starting materials were obtained from commercial sources and were used as received. Solvents were of analytical grade. Compound $\mathbf{1}$ was synthesized as described elsewhere (Rudolph et al., 2016). Thin-layer chromatography (TLC) was performed on Silica gel $60 \mathrm{~F}_{254}$ TLC plates (Merck KGaA, Darmstadt). The reported $R_{\mathrm{F}}$ values are uncorrected. Flash chromatography was carried out with a $40 \mathrm{~g}$ puriFlash column (30 $\mu \mathrm{m}$ silica gel, $60 \AA, 500 \mathrm{~m}^{2} \mathrm{~g}^{-1}$, Interchim, Montluçon, France). Preparative HPLC was performed on a Shimadzu LC-10AD system using $19 \times 150 \mathrm{~mm}$ XTerra RP-18 columns (7 $\mu \mathrm{m}$, Waters, Milford, Massachusetts, USA). ${ }^{1} \mathrm{H}$ and ${ }^{13} \mathrm{C}$
NMR spectra were recorded at room temperature on an Agilent Technologies VNMRS $400 \mathrm{MHz}$ NMR spectrometer ( $b s=$ broad singlet, $q=$ quartet and $m=$ multiplet). Chemical shifts are referenced to the residual signals of $\mathrm{CDCl}_{3}\left(\delta_{\mathrm{H}}=\right.$ $7.26 \mathrm{ppm}$ and $\left.\delta_{\mathrm{C}}=77.0 \mathrm{ppm}\right)$. High-resolution mass spectra (HRMS) were measured on a Bruker Daltonics APEXIII FTICR mass spectrometer.

\subsection{Synthesis and crystallization}

Compounds $\mathbf{2}$ and $\mathbf{3}$ were obtained when $\mathbf{1}$ was treated with 3-chloroperbenzoic acid, adapting the procedure described by Tiwari et al. (2015). A solution of 3-chloroperbenzoic acid $(1.04 \mathrm{~g}, 6.0 \mathrm{mmol})$ in dichloromethane $(6.5 \mathrm{ml})$ was added dropwise to a stirred solution of 8-nitro-2-(piperidin-1-yl)-6-(trifluoromethyl)-4H-1,3-benzothiazin-4-one, $\mathbf{1}(1.09 \mathrm{~g}, 3.0 \mathrm{mmol})$, in dichloromethane $(5 \mathrm{ml})$ at $0{ }^{\circ} \mathrm{C}$. After stirring for $4 \mathrm{~d}$ at room temperature, additional 3 -chloroperbenzoic acid $(0.5 \mathrm{~g})$ was added and the mixture was stirred for another day. The resulting mixture was washed twice with a saturated sodium bicarbonate solution $(55 \mathrm{ml})$ and then once with deionized water $(55 \mathrm{ml})$. After drying over sodium sulfate, the solvent was removed using a rotary evaporator. The crude product was subjected to flash chromatography [gradient of 50-100 $(v / v)$ ethyl acetate/heptane] to give $\mathbf{2}$ and $\mathbf{3}$. Both compounds were purified by HPLC [gradient of 5-95 $(v / v)$ acetonitrile/ water in $10 \mathrm{~min}+0.05 \%$ trifluoroacetic acid] to yield $6 \mathrm{mg}$ of $\mathbf{2}$ ( $0.016 \mathrm{mmol}, 0.5 \%)$ and $35 \mathrm{mg}$ of $\mathbf{3}(0.089 \mathrm{mmol}, 3 \%)$.

Crystals of $\mathbf{3}$ suitable for single-crystal X-ray analysis were obtained after a couple of days when a solution of $c a 5 \mathrm{mg}$ of the compound in ethanol $(1.5 \mathrm{ml})$ in a $10 \times 50 \mathrm{~mm}$ glass vial with a screw cap was left at room temperature and the solvent allowed to evaporate slowly.

The synthesis of $\mathbf{4}$ has been reported elsewhere (Tiwari et al., 2015; therein mistaken for the sulfone of the BTZ043 starting material). For the preparation of crystals suitable for single-crystal X-ray analysis, the compound $(1 \mathrm{mg})$ was added

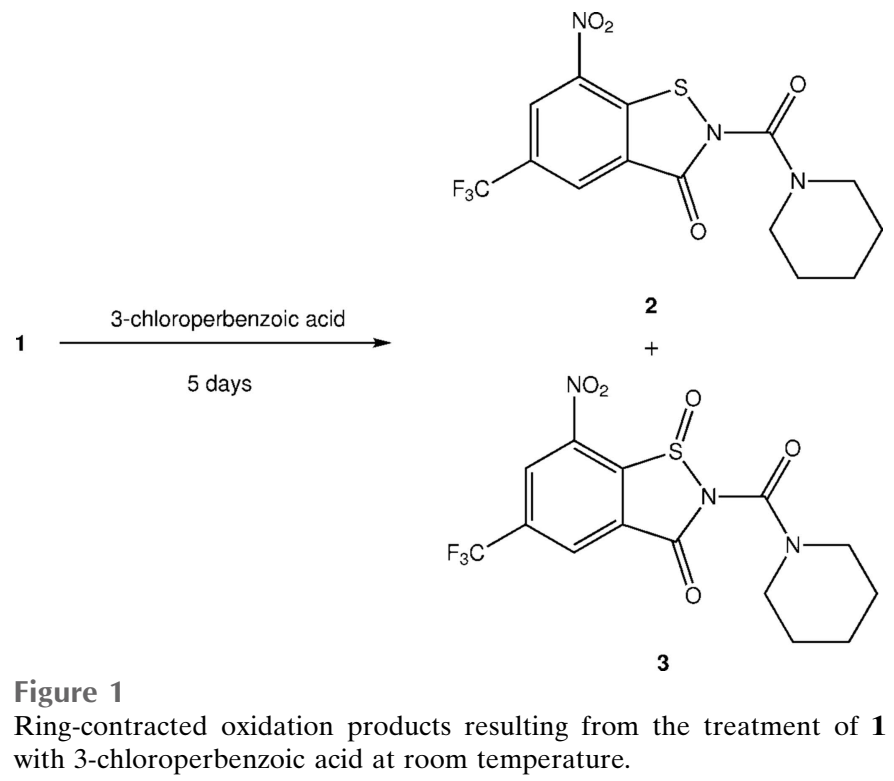


Table 1

Experimental details.

Experiments were carried out with $\mathrm{Cu} K \alpha$ radiation. Refinement in both cases was with 1 restraint. $\mathrm{H}$-atom parameters were constrained.

\begin{tabular}{|c|c|c|}
\hline & 3 & 4 \\
\hline Chemical formula & $\mathrm{C}_{14} \mathrm{H}_{12} \mathrm{~F}_{3} \mathrm{~N}_{3} \mathrm{O}_{5} \mathrm{~S}$ & $\mathrm{C}_{17} \mathrm{H}_{16} \mathrm{~F}_{3} \mathrm{~N}_{3} \mathrm{O}_{7} \mathrm{~S}$ \\
\hline$M_{\mathrm{r}}$ & 391.33 & 463.39 \\
\hline Temperature $(\mathrm{K})$ & 100 & 120 \\
\hline$a, b, c(\AA)$ & $17.6719(8), 25.7296(12), 6.8887$ (3) & $8.8165(2), 16.1649(4), 13.4246(3)$ \\
\hline$\alpha, \beta, \gamma\left({ }^{\circ}\right)$ & $90,90,90$ & $90,90.0022(12), 90$ \\
\hline Crystal size $(\mathrm{mm})$ & $0.23 \times 0.06 \times 0.04$ & $0.25 \times 0.07 \times 0.04$ \\
\hline \multicolumn{3}{|l|}{ Data collection } \\
\hline Diffractometer & Bruker Kappa Mach3 APEXII & Bruker PHOTON-II \\
\hline Absorption correction & Gaussian ( $S A D A B S ;$ Bruker, 2012) & Numerical (SADABS; Bruker, 2012) \\
\hline$T_{\min }, T_{\max }$ & $0.748,0.936$ & $0.715,0.949$ \\
\hline \multicolumn{3}{|l|}{ Refinement } \\
\hline$R\left[F^{2}>2 \sigma\left(F^{2}\right)\right], w R\left(F^{2}\right), S$ & $0.059,0.170,1.08$ & $0.033,0.083,1.04$ \\
\hline No. of reflections & 2477 & 7140 \\
\hline No. of parameters & 235 & 561 \\
\hline$\Delta \rho_{\max }, \Delta \rho_{\min }\left(\mathrm{e} \AA^{-3}\right)$ & $0.53,-0.75$ & $0.61,-0.31$ \\
\hline Absolute structure & $\begin{array}{l}\text { Flack } x \text { determined using } 550 \text { quotients } \\
\qquad\left[\left(I^{+}\right)-\left(I^{-}\right)\right] /\left[\left(I^{+}\right)+\left(I^{-}\right)\right](\text {Parsons } \text { et al., 2013) }\end{array}$ & $\begin{array}{l}\text { Flack } x \text { determined using } 3080 \text { quotients } \\
\qquad\left[\left(I^{+}\right)-\left(I^{-}\right)\right] /\left[\left(I^{+}\right)+\left(I^{-}\right)\right] \text {(Parsons } \text { et al., 2013) }\end{array}$ \\
\hline Absolute structure parameter & $0.08(5)$ & $0.017(6)$ \\
\hline
\end{tabular}

Computer programs: APEX3 (Bruker, 2015), SAINT (Bruker, 2015), SHELXT (Sheldrick, 2015a), SHELXL2018 (Sheldrick, 2015b), DIAMOND (Brandenburg, 2018), enCIFer (Allen et al., 2004) and publCIF (Westrip, 2010).

to a $6 \times 50 \mathrm{~mm}$ round-bottomed borosilicate glass culture tube, and dissolved in chloroform $(0.4 \mathrm{ml})$ to give a clear homogenous solution. The tube was placed in a $20 \mathrm{ml}$ scintillation vial, followed by the addition of pentane $(5 \mathrm{ml})$. The vial was capped tightly and the resulting diffusion chamber was allowed to stand undisturbed at room temperature. After several days, crystals suitable for $\mathrm{X}$-ray analysis formed.

2.2.1. Analytical data for $2 .{ }^{1} \mathrm{H}$ NMR $\left(400 \mathrm{MHz}, \mathrm{CDCl}_{3}\right): \delta$ $8.77(b s, 1 \mathrm{H}), 8.57(b s, 1 \mathrm{H}), 3.58(m, 4 \mathrm{H}), 1.78-1.70(m, 6 \mathrm{H})$ ppm; HRMS(ESI): calculated for $\mathrm{C}_{14} \mathrm{H}_{12} \mathrm{~F}_{3} \mathrm{~N}_{3} \mathrm{O}_{4} \mathrm{~S}[M+\mathrm{Na}]^{+}$ 398.0398, found 398.0397; $R_{\mathrm{F}}=0.29$ (ethyl acetate/heptane, $2: 8 v / v)$.

2.2.2. Analytical data for $3 .{ }^{1} \mathrm{H} \mathrm{NMR}\left(400 \mathrm{MHz}, \mathrm{CDCl}_{3}\right) \delta$ $8.79(b s, 1 \mathrm{H}), 8.58(b s, 1 \mathrm{H}), 3.68-3.51(m, 4 \mathrm{H}), 1.81-1.62(m$, $6 \mathrm{H}) \mathrm{ppm} ;{ }^{13} \mathrm{C}$ NMR $\left(101 \mathrm{MHz}, \mathrm{CDCl}_{3}\right): \delta 159.5,148.3,144.8$, $143.0,137.8\left(q,{ }^{2} J_{\mathrm{C}, \mathrm{F}}=35.5 \mathrm{~Hz}\right), 132.8,129.4\left(q,{ }^{3} J_{\mathrm{C}, \mathrm{F}}=3.6 \mathrm{~Hz}\right)$, $126.6\left(q,{ }^{3} J_{\mathrm{C}, \mathrm{F}}=3.6 \mathrm{~Hz}\right), 121.6\left(q,{ }^{1} J_{\mathrm{C}, \mathrm{F}}=274.2 \mathrm{~Hz}\right), 47.5,25.8$, $24.0 \mathrm{ppm}$; HRMS(ESI): calculated for $\mathrm{C}_{14} \mathrm{H}_{12} \mathrm{~F}_{3} \mathrm{~N}_{3} \mathrm{O}_{5} \mathrm{~S}[M+\mathrm{H}]^{+}$ 392.0528, found 392.0526; $R_{\mathrm{F}}=0.22$ (ethyl acetate/heptane, $2: 8 v / v)$.

\subsection{Refinement}

Crystal data, data collection and structure refinement details are summarized in Table 1 . $\mathrm{H}$-atom positions were calculated geometrically, with aromatic $\mathrm{C}-\mathrm{H}=0.95 \AA$, methyl $\mathrm{C}-\mathrm{H}=0.98 \AA$, methylene $\mathrm{C}-\mathrm{H}=0.99 \AA$ and methine
$\mathrm{C}-\mathrm{H}=1.00 \AA$, and refined using a riding model, with $U_{\text {iso }}(\mathrm{H})=1.2 U_{\text {eq }}(\mathrm{C})(1.5$ for methyl groups). The torsion angles of the methyl groups were initially determined using a circular Fourier search and subsequently refined while maintaining the tetrahedral structure.

\section{Results and discussion}

\subsection{Synthesis and structural identification}

When 1 was treated with 3-chloroperbenzoic acid, adapting the procedure for BTZ043 reported by Tiwari et al. (2015), likewise a major amount of the BTZ starting material was recovered, but small quantities of oxidation products $\mathbf{2}$ and $\mathbf{3}$ could be isolated by chromatography. The corresponding sum formulae were obtained from high-resolution mass spectra, and $\mathbf{3}$ was subjected to X-ray crystallography. The X-ray analysis umambiguously revealed the BIT 1-oxide structure for $\mathbf{3}$ instead of the anticipated BTZ sulfone, which would be a constitutional isomer (Fig. 1). Accordingly, and in agreement with the sum formula, we propose the corresponding BIT structure for $\mathbf{2}$ instead of the anticipated BTZ sulfoxide, which likewise would be a constitutional isomer.

Single-crystal X-ray analysis of the oxidation product of BTZ043 resulting from treatment with 3-chloroperbenzoic acid, which was named 'BTZ-SO ${ }_{2}$ ' by Tiwari et al. (2015), provided clear evidence for the formation of the corre- 
Table 2

${ }^{1} \mathrm{H}$ NMR shifts (ppm) of the aromatic protons in $\mathrm{CDCl}_{3}$ for $\mathbf{1}-\mathbf{3}, \mathrm{BTZ} 043$ and its oxidation products.

Data for 1 were taken from Rudolph et al. (2016) and data for BTZ043, 'BTZSO' and 'BTZ-SO ${ }_{2}$ ' were taken from Tiwari et al. (2015).

\begin{tabular}{llllll}
\hline $\mathbf{1}$ & $\mathbf{2}$ & $\mathbf{3}$ & $\mathrm{BTZ043}$ & 'BTZ-SO' & 'BTZ-SO $_{2}{ }^{\prime}(\mathbf{4})$ \\
\hline 9.08 & 8.77 & 8.79 & 9.02 & 8.78 & 8.80 \\
8.72 & 8.57 & 8.58 & 8.55 & 8.59 & 8.58
\end{tabular}

sponding BIT 1-oxide 4, a constitutional isomer of the reported BTZ sulfone (Fig. 2).

Table 2 compares the ${ }^{1} \mathrm{H}$ NMR shifts of the two aromatic protons in 1 and BTZ043 with those of the derived oxidation products. For both $\mathbf{2}$ and 3, as well as 'BTZ-SO' and 4, the signals assigned to the two aromatic protons are upfield shifted compared with the parent BTZs. While assuming the anticipated BTZ sulfoxide and sulfone structures, Tiwari et al. (2015) attributed this effect to the influence of the S-atom lone-pair delocalization and the loss of aromaticity due to the nonplanarity of the 1,4-thiazinone rings in the assumed BTZ sulfoxide and sulfone structures. Higher electron density within the encountered BIT nine-membered heterobicyclic system, as compared with the BTZ ten-membered system, however, provides a better explanation for the shielding of the aromatic protons resulting in the observed upfield shifts. For further corroboration, BIT 2, for which we did not obtain crystals suitable for single-crystal X-ray analysis, was synthesized deliberately from 2-chloro-3-nitro-5-(trifluoromethyl)nitrobenzamide (see supporting information), following an established procedure for related BITs (Bhakuni et al., 2012). NMR spectroscopic and mass spectrometric data of the<smiles>C[C@H]1COC2(CCN(C3=NC(=O)c4cc(C(F)(F)F)cc([N+](=O)[O-])c4S3(=O)=O)CC2)O1</smiles><smiles>C[C@H]1COC2(CCN(C(=O)N3C(=O)c4cc(C(F)(F)F)cc([N+](=O)[O-])c4S3=O)CC2)O1</smiles>

Figure 2

4

The incorrect structure 'BTZ-SO ${ }_{2}$ ' (Tiwari et al., 2015) and the revised structure of 4, resulting from treatment of BTZ043 with 3-chloroperbenzoic acid at room temperature. product thus obtained agreed with those for $\mathbf{2}$ resulting from treatment of $\mathbf{1}$ with 3-chloroperbenzoic acid.

\subsection{Structural descriptions of 3 and 4}

Compound $\mathbf{3}$ crystallizes in the polar orthorhombic space group $I b a 2$ with one molecule in the asymmetric unit $\left(Z^{\prime}=1\right)$. Fig. 3 shows the molecular structure in the crystal. The BIT system is not entirely planar. Atoms $\mathrm{N} 2$ and $\mathrm{O} 2$ are displaced by 0.27 (1) and -0.20 (1) $\AA$, respectively, above and below the mean plane defined by the benzene ring. The plane of the nitro group is tilted out of this plane by $12(1)^{\circ}$. The sulfinamide moiety exhibits a pyramidal structure at the $\mathrm{S}$ atom, as expected. The molecule in the chosen asymmetric unit is $R$ configured at the $\mathrm{S}$ atom. It is worth emphasizing, however, that the $S$ enantiomer is generated by glide symmetry in the polar crystal structure so the crystal is a racemate. The central carbamide moiety is tilted out of the BIT plane, as revealed by the torsion angles about the $\mathrm{N} 2-\mathrm{C} 9$ bond. The structure at atom N2 is slightly pyramidal, whereas that at N4 is virtually planar due to conjugation with the adjacent carbonyl group. The piperidine ring adopts a low-energy chair conformation with some deviations of the bond angles from ideal tetrahedal angles, which can be attributed to the planarity at N4.

Compound 4 crystallizes in the Sohncke space group $P 2_{1}$ with two diastereomers in the asymmetric unit $\left(Z^{\prime}=2\right)$. Fig. 4 depicts displacement ellipsoid plots for both unique molecules. Compared with $\mathbf{3}$, compound $\mathbf{4}$ exhibits an additional spiro(2S)-methyl-1,3-dioxolane group appended to the piperidine ring in the 4-position. The $S$ configuration at $\mathrm{C} 15$, as in the BTZ043 starting material, is encountered in both crystallographically distinct molecules and the configurational assignment was confirmed by a Flack $x$ parameter (Parsons et al., 2013) close to zero with a reasonably small standard uncertainty (Table 1). The two independent molecules exhibit opposite configurations at the $\mathrm{S}$ atoms and thus the crystal is a cocrystal of two diastereomers. Possible causes of $Z^{\prime}>1$ crystallization have been discussed (Steed \& Steed, 2015). Here, $Z^{\prime}=2$ is attributed to diasteromeric crystallization. The formation of a diastereomeric conglomerate or a solid solution

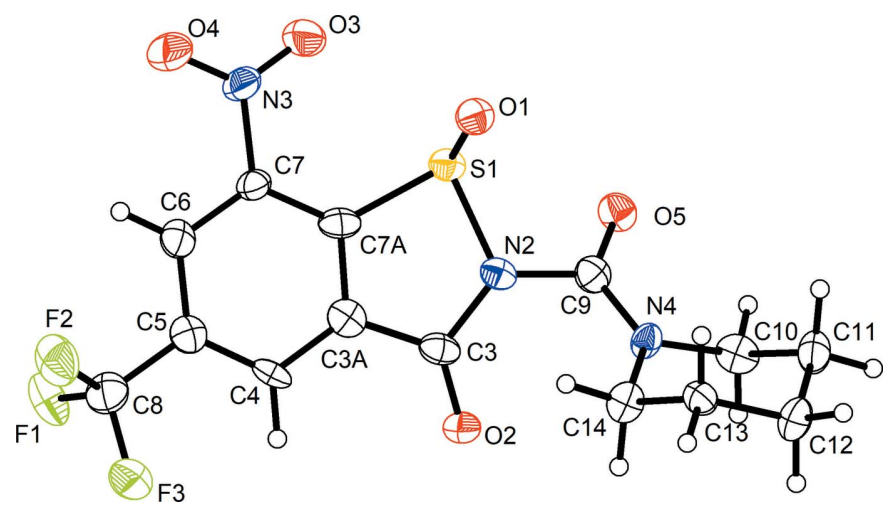

Figure 3

The molecular structure of $\mathbf{3}$ in the crystal. Displacement ellipsoids are drawn at the $50 \%$ probability level. $\mathrm{H}$ atoms are represented by small spheres of arbitrary radii. 


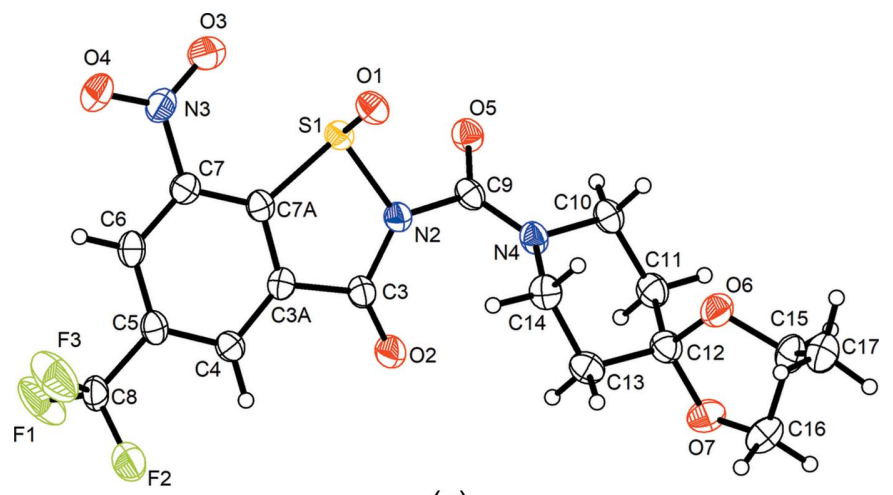

(a)

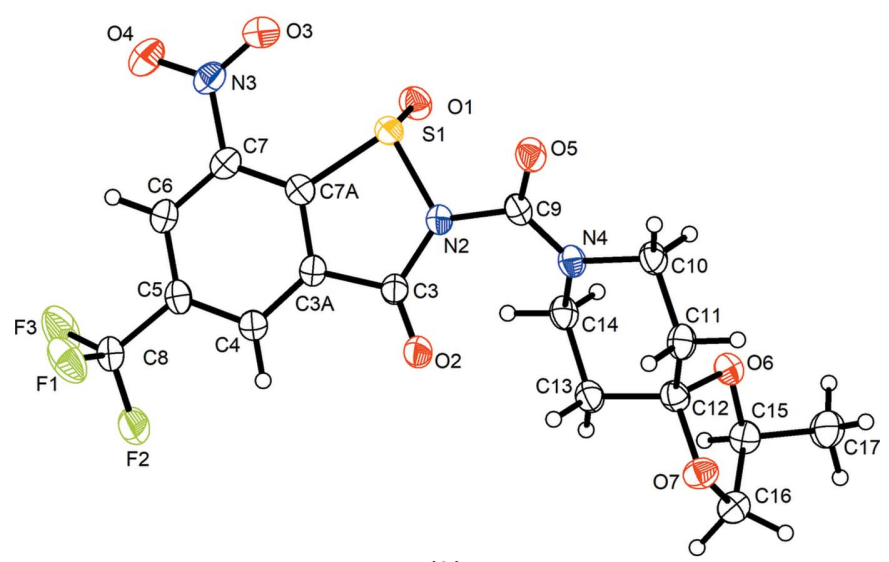

Figure 4

(b)

Displacement ellipsoid plots (50\% probability level) of the two crystallographically distinct diastereomeric molecules of $4 . \mathrm{H}$ atoms are represented by small spheres of arbitrary radii.

would have been an alternative crystallization pathway. Apart from the configuration at the $\mathrm{S}$ atom, the distinct molecules also exhibit different conformations of the 1,3-dioxolane fivemembered rings. In molecule 1 (Fig. $4 a$ ), the 1,3-dioxolane ring adopts an envelope conformation with atom $\mathrm{C} 16$ on the flap, whereas in molecule 2 (Fig. $4 b$ ), the ring is close to an envelope with the spiro atom $\mathrm{C} 12$ on the flap. As in $\mathbf{3}$, the BIT systems deviate slightly from planarity. In molecule 1 , atom $\mathrm{O} 2$ is displaced from the mean plane of the benzene ring by 0.323 (6) $\AA$, and in molecule 2, atoms N2 and O2 deviate by -0.118 (5) and 0.333 (5) $\AA$, respectively, from this plane. The tilt angle between the mean plane of the benzene ring and the plane of the nitro group is $16.4(3)^{\circ}$ in molecule 1 and $10.7(4)^{\circ}$ in molecule 2. Similar to $\mathbf{3}$, in both molecules, the central carbamide moiety is tilted out of the plane of the BIT skeleton and the appended piperidine ring adopts a low-energy chair conformation with some minor deviations of the bond angles.

The supramolecular structure of $\mathbf{4}$ in the solid state features short $\mathrm{C}-\mathrm{F} \cdot \mathrm{F}-\mathrm{F}-\mathrm{C}$ contacts $\left[\mathrm{F} 1 \_1 \cdots \mathrm{F} 32^{\mathrm{i}}=2.737\right.$ (4) $\AA$ and $\left.\left.\mathrm{F} 3 \_1 \cdots \mathrm{F} 1 \_2^{\mathrm{ii}}=2.751(4) \AA\right)\right]$, which link unique molecules 1 and 2 along the [100] direction (Fig. 5). According to the corresponding $\mathrm{C}-\mathrm{F} \cdots \mathrm{F}$ angles in the range of $157.8-167.8^{\circ}$, these contacts may be classified as type-I F. . F interactions (Baker et al., 2012). F...F contacts that are shorter than the sum of the van der Waals radii are not encountered in the crystal structure of $\mathbf{3}$, but instead several short $\mathrm{C}-\mathrm{H} \cdots \mathrm{F}$ contacts are observed (not depicted).

\subsection{Mechanistic discussion of the ring contraction}

Since the ring-contracted oxidation products only formed in very low yields, investigation of the reaction mechanism of BTZ oxidation and rearrangement upon treatment with 3-chloroperbenzoic acid was not undertaken. We propose the sequence shown in Fig. 6. This is in part based on a mechanism

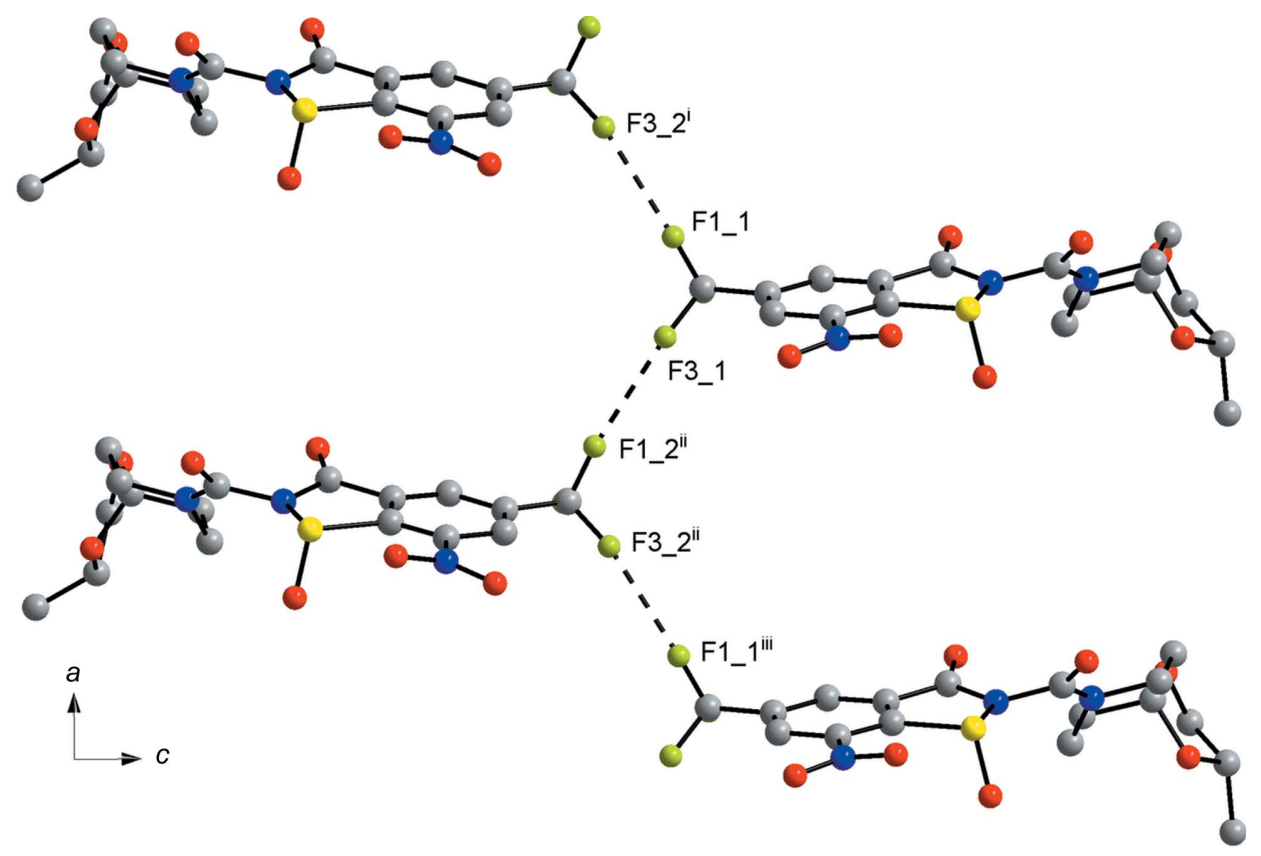

Figure 5

Part of the crystal structure of 4 , showing $\mathrm{C}-\mathrm{F} \cdots \mathrm{F}-\mathrm{C}$ contacts (dashed lines), viewed down the $b$-axis direction towards the origin. [Symmetry codes: (i) $x, y, z-1$; (ii) $x-1, y, z-1$; (iii) $x-1, y, z$.] 
Table 3

In vitro activities $\left(\mathrm{MIC}_{90}\right.$ in $\left.\mu M\right)$ of $\mathbf{1 - 3}, \mathrm{BTZ} 043$ and its oxidation products.

Data for BTZ043, 'BTZ-SO' and 'BTZ-SO ${ }_{2}$ ' were taken from Tiwari et al. (2015).

\begin{tabular}{lllllll}
\hline & $\mathbf{1}$ & $\mathbf{2}$ & $\mathbf{3}$ & \multicolumn{1}{c}{ BTZ043 } & 'BTZ-SO' & 'BTZ-SO ${ }_{2}$ ' (4) \\
\hline M. tuberculosis & $4.3^{a}$ & $<0.26^{a}$ & $8.0^{a}$ & $0.02^{b}$ & $0.06^{b}$ & $0.48^{b}$ \\
M. aurum & $10.9^{c}$ & $2.0^{c}$ & $19.4^{c}$ & $>200^{a}$ & $3.13-12.5^{d}$ & $>200^{d}$ \\
\hline
\end{tabular}

Notes: (a) M. tuberculosis $\mathrm{H}_{37} \mathrm{Rv}$ pTEC27 (7H9 medium plus $10 \%$ OADC and $0.05 \%$ polysorbate 80, microplate RFP assay); (b) M. tuberculosis $\mathrm{H}_{37} \mathrm{Rv}$ (7H9 medium plus casitone, palmitic acid, albumin and catalase; MABA, Microplate Alamar Blue Assay); (c) M. aurum DSMZ 43999 (7H9 medium plus 10\% OADC and $0.5 \%$ glycerol, microplate OD600 Assay); (d) M. aurum SB66.

postulated by Szabó et al. (1988). We follow these authors in assuming that the anticipated oxidation of $\mathbf{1}$ to the corresponding BTZ sulfoxide occurred initially and was followed by nucleophilic addition of water (from wet 3-chloroperbenzoic acid used) to the $\mathrm{C}=\mathrm{N}$ bond of the BTZ system. Ring opening and rearrangement to a sulfenic acid group and an $N$-acylcarbamide moiety within the molecule would be followed by the loss of water to form $\mathbf{2}$, which was then oxidized by another equivalent of 3-chloroperbenzoic acid leading to $\mathbf{3}$, which we isolated and structurally characterized by X-ray crystallography. Although this mechanism is only postulated, it explains why both $\mathbf{2}$ and $\mathbf{3}$ were formed.

\subsection{Antimycobacterial activities}

Tiwari et al. (2015) reported in vitro activities of the oxidation products against Mycobacterium tuberculosis and $M$. aurum, among other mycobacteria, albeit assuming the BTZ sulfoxide and sulfone structures, which are revised in the present work. We also evaluated the activities of $\mathbf{2}$ and $\mathbf{3}$ against M. tuberculosis and M. aurum (the assay protocols can be found in the supporting information). Although the structures of $\mathbf{2}$ and $\mathbf{3}$ differ from those of 'BTZ-SO' and $\mathbf{4}$ by the absence of the spiro-(2S)-methyl-1,3-dioxolane group appended to the piperidine ring in the 4-position, their activities against $M$. tuberculosis and M. aurum are comparable (Table 3). Indeed, BITs are known to have antimicrobial activity and are used as preservatives (Novick et al., 2013). Interestingly, BIT $\mathbf{2}$ and its 1-oxide $\mathbf{3}$, as well as 'BTZSO' and $\mathbf{4}$, show comparable or better activity against both mycobacterial species than the corresponding BTZs $\mathbf{1}$ and BTZ043 (Table 3). Thus, BITs could likewise be considered as decaprenylphosphoryl- $\beta$-D-ribose $2^{\prime}$-epimerase (DprE1) inhibitors, and work along this line is in progess. It should be noted, however, that BITs are known to have various molecular targets in microorganisms (Gopinath et al., 2017). This may render them less promising for the development of antimycobacterial agents.

\section{Acknowledgements}

We thank Dr Matthias Schmidt for performing the preparative HPLC of $\mathbf{2}$ and $\mathbf{3}$ and Nils Nöthling for the X-ray intensity data collection for 3. Professor Christian W. Lehmann is gratefully acknowledged for providing access to the X-ray diffraction facility used to collect the data for 3. AR and HAS would like to thank Professor Yossef Av-Gay for his support. Open access funding enabled and organized by Projekt DEAL.

\section{References}

Allen, F. H., Johnson, O., Shields, G. P., Smith, B. R. \& Towler, M. (2004). J. Appl. Cryst. 37, 335-338.

Baker, R. J., Colavita, P. E., Murphy, D. M., Platts, J. A. \& Wallis, J. D. (2012). J. Phys. Chem. A, 116, 1435-1444.

Bhakuni, B. S., Balkrishna, S. J., Kumar, A. \& Kumar, S. (2012). Tetrahedron Lett. 53, 1354-1357.

Brandenburg, K. (2018). DIAMOND. Version 3.2k. Crystal Impact GbR, Bonn, Germany.

Bruker (2012). SADABS. Bruker AXS Inc., Madison, Wisconsin, USA.

Bruker (2015). APEX3 and SAINT. Bruker AXS Inc., Madison, Wisconsin, USA.<smiles>O=C1NC(O)(N2CCCCC2)S(=O)(=O)c2c1cc(C(F)(F)F)cc2[N+](=O)[O-]</smiles>

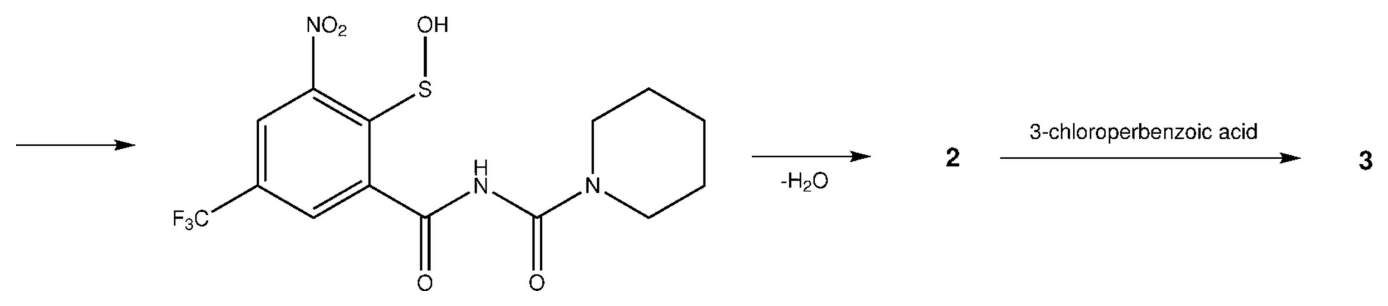

Figure 6

Postulated reaction mechanism for the formation of BITs and BIT 1-oxides from BTZs upon treatment with 3-chloroperbenzoic acid (shown for 1). 
Gopinath, P., Yadav, R. K., Shukla, P. K., Srivastava, K., Puri, S. K. \& Muraleedharan, K. M. (2017). Bioorg. Med. Chem. Lett. 27, 12911295.

Kloss, F., Krchnak, V., Krchnakova, A., Schieferdecker, S., Dreisbach, J., Krone, V., Möllmann, U., Hoelscher, M. \& Miller, M. J. (2017). Angew. Chem. Int. Ed. 56, 2187-2191.

Lupien, A., Vocat, A., Foo, C. S.-Y., Blattes, E., Gillon, J.-Y., Makarov, V. \& Cole, S. T. (2018). Antimicrob. Agents Chemother. 62, e0084018.

Lv, K., You, X., Wang, B., Wei, Z., Chai, Y., Wang, B., Wang, A., Huang, G., Liu, M. \& Lu, Y. (2017). ACS Med. Chem. Lett. 8, 636641.

Makarov, V. \& Mikušová, K. (2020). Appl. Sci. 10, 2269.

Mikušová, K., Makarov, V. \& Neres, J. (2014). Curr. Pharm. Des. 20, 4379-4403.

Novick, R. M., Nelson, M. L., Unice, K. M., Keenan, J. J. \& Paustenbach, D. J. (2013). Food Chem. Toxicol. 56, 60-66.
Parsons, S., Flack, H. D. \& Wagner, T. (2013). Acta Cryst. B69, 249-259.

Rudolph, I., Imming, P. \& Richter, A. (2016). Ger. Offen. DE 102014012546 A1 20160331.

Sheldrick, G. M. (2015a). Acta Cryst. A71, 3-8.

Sheldrick, G. M. (2015b). Acta Cryst. C71, 3-8.

Steed, K. M. \& Steed, J. W. (2015). Chem. Rev. 115, 2895-2933.

Szabó, J., Szücs, E., Fodor, L., Katócs, Á., Bernáth, G. \& Sohár, P. (1988). Tetrahedron, 44, 2985-2992.

Tiwari, R., Miller, P. A., Cho, S., Franzblau, S. G. \& Miller, M. J. (2015). ACS Med. Chem. Lett. 6, 128-133.

Tiwari, R., Moraski, G. C., Krchňák, V., Miller, P. A., Colon-Martinez, M., Herrero, E., Oliver, A. G. \& Miller, M. J. (2013). J. Am. Chem. Soc. 135, 3539-3549.

Westrip, S. P. (2010). J. Appl. Cryst. 43, 920-925.

Zhang, G. \& Aldrich, C. C. (2019). Acta Cryst. C75, 1031-1035.

Zhang, G., Howe, M. \& Aldrich, C. A. (2019). ACS Med. Chem. Lett. 10, 348-351. 


\section{supporting information}

Acta Cryst. (2020). C76, 907-913 [https://doi.org/10.1107/S2053229620010931]

\section{Crystallographic evidence for unintended benzisothiazolinone 1-oxide}

\section{formation from benzothiazinones through oxidation}

Tamira Eckhardt, Richard Goddard, Christoph Lehmann, Adrian Richter, Henok Asfaw Sahile, Rui Liu, Rohit Tiwari, Allen G. Oliver, Marvin J. Miller, Rüdiger W. Seidel and Peter Imming

Computing details

For both structures, data collection: APEX3 (Bruker, 2015); cell refinement: SAINT (Bruker, 2015); data reduction: SAINT (Bruker, 2015); program(s) used to solve structure: SHELXT (Sheldrick, 2015a); program(s) used to refine structure: SHELXL2018 (Sheldrick, 2015b); molecular graphics: DIAMOND (Brandenburg, 2018); software used to prepare material for publication: enCIFer (Allen et al., 2004) and publCIF (Westrip, 2010).

7-Nitro-2-(piperidine-1-carbonyl)-5-(trifluoromethyl)benzo[d] isothiazol-3(2H)-one 1-oxide (3)

Crystal data

$\mathrm{C}_{14} \mathrm{H}_{12} \mathrm{~F}_{3} \mathrm{~N}_{3} \mathrm{O}_{5} \mathrm{~S}$

$M_{r}=391.33$

Orthorhombic, Iba2

$a=17.6719(8) \AA$

$b=25.7296(12) \AA$

$c=6.8887(3) \AA$

$V=3132.2(2) \AA^{3}$

$Z=8$

$F(000)=1600$

\section{Data collection}

Bruker Kappa Mach3 APEXII diffractometer

Radiation source: $0.2 \times 2 \mathrm{~mm}^{2}$ focus rotating anode

MONTEL graded multilayer optics monochromator

Detector resolution: 66.67 pixels $\mathrm{mm}^{-1}$

$\varphi$ - and $\omega$-scans

Absorption correction: gaussian

(SADABS; Bruker, 2012)

Refinement

Refinement on $F^{2}$

Least-squares matrix: full

$R\left[F^{2}>2 \sigma\left(F^{2}\right)\right]=0.059$

$w R\left(F^{2}\right)=0.170$

$S=1.08$

2477 reflections

235 parameters
$D_{\mathrm{x}}=1.660 \mathrm{Mg} \mathrm{m}^{-3}$

$\mathrm{Cu} K \alpha$ radiation, $\lambda=1.54178 \AA$

Cell parameters from 2591 reflections

$\theta=3.0-47.9^{\circ}$

$\mu=2.50 \mathrm{~mm}^{-1}$

$T=100 \mathrm{~K}$

Needle, yellow

$0.23 \times 0.06 \times 0.04 \mathrm{~mm}$

$T_{\min }=0.748, T_{\max }=0.936$

22457 measured reflections

2477 independent reflections

1843 reflections with $I>2 \sigma(I)$

$R_{\text {int }}=0.102$

$\theta_{\max }=70.1^{\circ}, \theta_{\min }=3.0^{\circ}$

$h=-20 \rightarrow 18$

$k=-29 \rightarrow 29$

$l=-7 \rightarrow 7$

1 restraint

Primary atom site location: dual

Secondary atom site location: difference Fourier map

Hydrogen site location: inferred from neighbouring sites

$\mathrm{H}$-atom parameters constrained 
$w=1 /\left[\sigma^{2}\left(F_{0}^{2}\right)+(0.0581 P)^{2}+17.2243 P\right]$

where $P=\left(F_{\mathrm{o}}^{2}+2 F_{\mathrm{c}}^{2}\right) / 3$

$(\Delta / \sigma)_{\max }<0.001$

$\Delta \rho_{\max }=0.53$ e $\AA^{-3}$

$\Delta \rho_{\min }=-0.75$ e $\AA^{-3}$
Absolute structure: Flack $x$ determined using 550 quotients $[(\mathrm{I}+)-(\mathrm{I}-)] /[(\mathrm{I}+)+(\mathrm{I}-)]$ (Parsons $e t$ al., 2013)

Absolute structure parameter: 0.08 (5)

\section{Special details}

Geometry. All esds (except the esd in the dihedral angle between two 1.s. planes) are estimated using the full covariance matrix. The cell esds are taken into account individually in the estimation of esds in distances, angles and torsion angles; correlations between esds in cell parameters are only used when they are defined by crystal symmetry. An approximate (isotropic) treatment of cell esds is used for estimating esds involving l.s. planes.

Fractional atomic coordinates and isotropic or equivalent isotropic displacement parameters $\left(\hat{A}^{2}\right)$

\begin{tabular}{|c|c|c|c|c|}
\hline & $x$ & $y$ & $z$ & $U_{\text {iso }} * / U_{\text {eq }}$ \\
\hline S1 & $0.39270(11)$ & $0.23902(8)$ & $0.2946(4)$ & $0.0263(5)$ \\
\hline $\mathrm{F} 1$ & $0.2738(3)$ & $0.4716(2)$ & $0.6178(10)$ & $0.0484(17)$ \\
\hline $\mathrm{F} 2$ & $0.3923(3)$ & $0.4791(2)$ & $0.6776(10)$ & $0.0498(17)$ \\
\hline F3 & $0.3204(4)$ & $0.4389(2)$ & $0.8789(9)$ & $0.0471(17)$ \\
\hline $\mathrm{O} 1$ & $0.4744(3)$ & $0.2295(2)$ & $0.2901(11)$ & $0.0306(14)$ \\
\hline $\mathrm{O} 2$ & $0.2793(3)$ & $0.2404(2)$ & $0.7694(9)$ & $0.0298(15)$ \\
\hline $\mathrm{O} 3$ & $0.4304(4)$ & $0.3133(3)$ & $0.0182(11)$ & $0.0428(18)$ \\
\hline O4 & $0.4590(4)$ & $0.3947(3)$ & $0.0606(12)$ & $0.0478(19)$ \\
\hline O5 & $0.3327(3)$ & $0.1386(2)$ & $0.3621(9)$ & $0.0308(16)$ \\
\hline N2 & $0.3585(4)$ & $0.2155(3)$ & $0.5159(11)$ & $0.0242(17)$ \\
\hline N3 & $0.4310(4)$ & $0.3531(3)$ & $0.1142(12)$ & $0.035(2)$ \\
\hline N4 & $0.3505(4)$ & $0.1367(3)$ & $0.6918(11)$ & $0.0244(16)$ \\
\hline $\mathrm{C} 3$ & $0.3214(5)$ & $0.2512(3)$ & $0.6345(14)$ & $0.027(2)$ \\
\hline $\mathrm{C} 3 \mathrm{~A}$ & $0.3407(4)$ & $0.3036(4)$ & $0.5686(14)$ & $0.027(2)$ \\
\hline $\mathrm{C} 4$ & $0.3244(5)$ & $0.3493(3)$ & $0.6639(14)$ & $0.027(2)$ \\
\hline $\mathrm{H} 4$ & 0.297101 & 0.348687 & 0.782699 & $0.033^{*}$ \\
\hline $\mathrm{C} 5$ & $0.3480(5)$ & $0.3961(4)$ & $0.5857(15)$ & $0.029(2)$ \\
\hline C6 & $0.3843(5)$ & 0.3979 (4) & $0.4055(15)$ & $0.030(2)$ \\
\hline H6 & 0.399673 & 0.430018 & 0.350254 & $0.036^{*}$ \\
\hline $\mathrm{C} 7$ & $0.3970(5)$ & $0.3513(3)$ & $0.3099(16)$ & $0.0262(19)$ \\
\hline C7A & $0.3775(5)$ & $0.3036(4)$ & $0.3912(14)$ & $0.028(2)$ \\
\hline $\mathrm{C} 8$ & $0.3337(6)$ & $0.4459(4)$ & $0.6897(18)$ & $0.038(3)$ \\
\hline C9 & $0.3460(5)$ & $0.1602(3)$ & $0.5197(15)$ & $0.029(2)$ \\
\hline $\mathrm{C} 10$ & $0.3233(5)$ & $0.0826(4)$ & $0.7055(15)$ & $0.032(2)$ \\
\hline H10A & 0.309365 & 0.070047 & 0.574432 & $0.039^{*}$ \\
\hline H10B & 0.277370 & 0.081524 & 0.787702 & $0.039^{*}$ \\
\hline C11 & $0.3836(5)$ & 0.0469 & $0.7919(18)$ & $0.034(2)$ \\
\hline H11A & 0.361581 & 0.011974 & 0.813779 & $0.041^{*}$ \\
\hline H11B & 0.425884 & 0.043181 & 0.698612 & $0.041^{*}$ \\
\hline $\mathrm{C} 12$ & $0.4138(6)$ & 0.0681 (4) & $0.9830(15)$ & $0.033(2)$ \\
\hline $\mathrm{H} 12 \mathrm{~A}$ & 0.455499 & 0.045824 & 1.030461 & $0.040^{*}$ \\
\hline H12B & 0.373036 & 0.068011 & 1.081686 & $0.040^{*}$ \\
\hline $\mathrm{C} 13$ & $0.4426(5)$ & 0.1238 & $0.9521(14)$ & $0.028(2)$ \\
\hline H13A & 0.460546 & 0.137967 & 1.077522 & $0.033^{*}$ \\
\hline
\end{tabular}


supporting information

\begin{tabular}{lllll} 
H13B & 0.486071 & 0.123146 & 0.861508 & $0.033^{*}$ \\
C14 & $0.3822(5)$ & $0.1587(4)$ & $0.8718(15)$ & $0.032(2)$ \\
H14A & 0.341411 & 0.162932 & 0.968949 & $0.039^{*}$ \\
H14B & 0.403903 & 0.193404 & 0.844520 & $0.039^{*}$ \\
\hline
\end{tabular}

Atomic displacement parameters $\left(\AA^{2}\right)$

\begin{tabular}{lllllll}
\hline & $U^{11}$ & $U^{22}$ & $U^{33}$ & $U^{12}$ & $U^{13}$ & $U^{23}$ \\
\hline S1 & $0.0261(10)$ & $0.0301(10)$ & $0.0225(11)$ & $0.0024(8)$ & $0.0004(10)$ & $-0.0012(11)$ \\
F1 & $0.045(3)$ & $0.035(3)$ & $0.065(5)$ & $0.014(3)$ & $-0.003(3)$ & $-0.012(3)$ \\
F2 & $0.047(4)$ & $0.035(3)$ & $0.067(5)$ & $-0.011(3)$ & $0.004(3)$ & $-0.011(3)$ \\
F3 & $0.062(4)$ & $0.038(3)$ & $0.040(4)$ & $-0.001(3)$ & $0.009(3)$ & $-0.009(3)$ \\
O1 & $0.026(3)$ & $0.036(3)$ & $0.030(4)$ & $0.006(2)$ & $0.001(3)$ & $0.004(3)$ \\
O2 & $0.031(3)$ & $0.032(3)$ & $0.027(4)$ & $0.002(3)$ & $0.008(3)$ & $0.001(3)$ \\
O3 & $0.055(5)$ & $0.045(5)$ & $0.029(4)$ & $0.013(4)$ & $0.005(4)$ & $0.001(3)$ \\
O4 & $0.047(4)$ & $0.049(4)$ & $0.048(5)$ & $0.003(4)$ & $0.021(4)$ & $0.010(4)$ \\
O5 & $0.033(4)$ & $0.032(4)$ & $0.027(4)$ & $0.001(3)$ & $-0.006(3)$ & $-0.004(3)$ \\
N2 & $0.021(4)$ & $0.029(4)$ & $0.022(4)$ & $0.002(3)$ & $-0.001(3)$ & $-0.004(3)$ \\
N3 & $0.035(5)$ & $0.036(5)$ & $0.034(5)$ & $0.006(4)$ & $0.012(4)$ & $0.011(4)$ \\
N4 & $0.033(4)$ & $0.022(4)$ & $0.019(4)$ & $-0.002(3)$ & $-0.007(3)$ & $0.002(3)$ \\
C3 & $0.020(5)$ & $0.038(5)$ & $0.024(6)$ & $0.005(4)$ & $0.000(4)$ & $-0.004(4)$ \\
C3A & $0.016(4)$ & $0.034(5)$ & $0.030(6)$ & $0.003(4)$ & $-0.007(4)$ & $-0.002(4)$ \\
C4 & $0.023(5)$ & $0.033(5)$ & $0.026(6)$ & $0.007(4)$ & $0.006(4)$ & $-0.011(4)$ \\
C5 & $0.022(4)$ & $0.030(5)$ & $0.033(6)$ & $0.001(4)$ & $-0.002(4)$ & $-0.001(4)$ \\
C6 & $0.020(4)$ & $0.029(5)$ & $0.040(6)$ & $0.004(4)$ & $0.004(4)$ & $0.004(4)$ \\
C7 & $0.025(4)$ & $0.028(4)$ & $0.025(5)$ & $0.002(4)$ & $0.006(4)$ & $0.005(5)$ \\
C7A & $0.016(4)$ & $0.041(6)$ & $0.026(5)$ & $0.000(4)$ & $0.005(4)$ & $0.000(4)$ \\
C8 & $0.031(5)$ & $0.039(6)$ & $0.045(8)$ & $-0.004(5)$ & $0.007(5)$ & $0.003(5)$ \\
C9 & $0.027(5)$ & $0.029(5)$ & $0.030(6)$ & $0.002(4)$ & $-0.001(4)$ & $-0.001(5)$ \\
C10 & $0.023(5)$ & $0.040(5)$ & $0.034(6)$ & $-0.004(4)$ & $-0.004(4)$ & $-0.006(4)$ \\
C11 & $0.038(5)$ & $0.025(5)$ & $0.041(6)$ & $0.002(4)$ & $-0.001(5)$ & $0.005(5)$ \\
C12 & $0.037(6)$ & $0.028(5)$ & $0.034(6)$ & $-0.001(4)$ & $-0.007(4)$ & $0.008(4)$ \\
C13 & $0.032(5)$ & $0.026(5)$ & $0.026(6)$ & $0.002(4)$ & $0.001(4)$ & $-0.003(4)$ \\
C14 & $0.031(5)$ & $0.031(5)$ & $0.034(6)$ & $-0.002(4)$ & $-0.004(5)$ & $0.003(4)$ \\
& & & & & & \\
\hline & & & & & &
\end{tabular}

Geometric parameters $\left(A,{ }^{\circ}\right)$

\begin{tabular}{llll}
\hline $\mathrm{S} 1-\mathrm{O} 1$ & $1.465(6)$ & $\mathrm{C} 4-\mathrm{H} 4$ & 0.9500 \\
$\mathrm{~S} 1-\mathrm{N} 2$ & $1.748(8)$ & $\mathrm{C} 5-\mathrm{C} 6$ & $1.398(14)$ \\
$\mathrm{S} 1-\mathrm{C} 7 \mathrm{~A}$ & $1.810(10)$ & $\mathrm{C} 5-\mathrm{C} 8$ & $1.490(14)$ \\
$\mathrm{F} 1-\mathrm{C} 8$ & $1.344(12)$ & $\mathrm{C} 6-\mathrm{C} 7$ & $1.386(13)$ \\
$\mathrm{F} 2-\mathrm{C} 8$ & $1.345(11)$ & $\mathrm{C} 6-\mathrm{H} 6$ & 0.9500 \\
$\mathrm{~F} 3-\mathrm{C} 8$ & $1.336(13)$ & $\mathrm{C} 7-\mathrm{C} 7 \mathrm{~A}$ & $1.393(12)$ \\
$\mathrm{O} 2-\mathrm{C} 3$ & $1.222(11)$ & $\mathrm{C} 10-\mathrm{C} 11$ & $1.529(13)$ \\
$\mathrm{O} 3-\mathrm{N} 3$ & $1.220(11)$ & $\mathrm{C} 10-\mathrm{H} 10 \mathrm{~A}$ & 0.9900 \\
$\mathrm{O} 4-\mathrm{N} 3$ & $1.236(10)$ & $\mathrm{C} 10-\mathrm{H} 10 \mathrm{~B}$ & 0.9900 \\
$\mathrm{O} 5-\mathrm{C} 9$ & $1.243(12)$ & $\mathrm{C} 11-\mathrm{C} 12$ & $1.522(15)$ \\
$\mathrm{N} 2-\mathrm{C} 3$ & $1.394(11)$ & $\mathrm{C} 11-\mathrm{H} 11 \mathrm{~A}$ & 0.9900
\end{tabular}




\begin{tabular}{|c|c|c|c|}
\hline $\mathrm{N} 2-\mathrm{C} 9$ & $1.439(11)$ & $\mathrm{C} 11-\mathrm{H} 11 \mathrm{~B}$ & 0.9900 \\
\hline $\mathrm{N} 3-\mathrm{C} 7$ & $1.477(13)$ & $\mathrm{C} 12-\mathrm{C} 13$ & $1.535(12)$ \\
\hline $\mathrm{N} 4-\mathrm{C} 9$ & $1.334(12)$ & $\mathrm{C} 12-\mathrm{H} 12 \mathrm{~A}$ & 0.9900 \\
\hline $\mathrm{N} 4-\mathrm{C} 14$ & $1.474(12)$ & $\mathrm{C} 12-\mathrm{H} 12 \mathrm{~B}$ & 0.9900 \\
\hline $\mathrm{N} 4-\mathrm{C} 10$ & $1.474(11)$ & $\mathrm{C} 13-\mathrm{C} 14$ & $1.500(12)$ \\
\hline $\mathrm{C} 3-\mathrm{C} 3 \mathrm{~A}$ & $1.461(13)$ & C13-H13A & 0.9900 \\
\hline $\mathrm{C} 3 \mathrm{~A}-\mathrm{C} 4$ & $1.378(12)$ & $\mathrm{C} 13-\mathrm{H} 13 \mathrm{~B}$ & 0.9900 \\
\hline $\mathrm{C} 3 \mathrm{~A}-\mathrm{C} 7 \mathrm{~A}$ & $1.384(14)$ & $\mathrm{C} 14-\mathrm{H} 14 \mathrm{~A}$ & 0.9900 \\
\hline $\mathrm{C} 4-\mathrm{C} 5$ & $1.384(12)$ & C14-H14B & 0.9900 \\
\hline $\mathrm{O} 1-\mathrm{S} 1-\mathrm{N} 2$ & $107.6(4)$ & $\mathrm{F} 3-\mathrm{C} 8-\mathrm{C} 5$ & $112.5(9)$ \\
\hline $\mathrm{O} 1-\mathrm{S} 1-\mathrm{C} 7 \mathrm{~A}$ & $107.9(4)$ & $\mathrm{F} 1-\mathrm{C} 8-\mathrm{C} 5$ & $112.4(9)$ \\
\hline $\mathrm{N} 2-\mathrm{S} 1-\mathrm{C} 7 \mathrm{~A}$ & $86.9(4)$ & $\mathrm{F} 2-\mathrm{C} 8-\mathrm{C} 5$ & $112.7(8)$ \\
\hline $\mathrm{C} 3-\mathrm{N} 2-\mathrm{C} 9$ & $124.7(8)$ & $\mathrm{O} 5-\mathrm{C} 9-\mathrm{N} 4$ & $125.7(8)$ \\
\hline $\mathrm{C} 3-\mathrm{N} 2-\mathrm{S} 1$ & $116.4(6)$ & $\mathrm{O} 5-\mathrm{C} 9-\mathrm{N} 2$ & $117.1(9)$ \\
\hline $\mathrm{C} 9-\mathrm{N} 2-\mathrm{S} 1$ & $114.3(6)$ & $\mathrm{N} 4-\mathrm{C} 9-\mathrm{N} 2$ & $117.1(8)$ \\
\hline $\mathrm{O} 3-\mathrm{N} 3-\mathrm{O} 4$ & $124.7(9)$ & $\mathrm{N} 4-\mathrm{C} 10-\mathrm{C} 11$ & $111.4(7)$ \\
\hline $\mathrm{O} 3-\mathrm{N} 3-\mathrm{C} 7$ & $117.7(8)$ & $\mathrm{N} 4-\mathrm{C} 10-\mathrm{H} 10 \mathrm{~A}$ & 109.3 \\
\hline $\mathrm{O} 4-\mathrm{N} 3-\mathrm{C} 7$ & $117.6(8)$ & $\mathrm{C} 11-\mathrm{C} 10-\mathrm{H} 10 \mathrm{~A}$ & 109.3 \\
\hline $\mathrm{C} 9-\mathrm{N} 4-\mathrm{C} 14$ & $126.6(7)$ & $\mathrm{N} 4-\mathrm{C} 10-\mathrm{H} 10 \mathrm{~B}$ & 109.3 \\
\hline $\mathrm{C} 9-\mathrm{N} 4-\mathrm{C} 10$ & $117.8(8)$ & $\mathrm{C} 11-\mathrm{C} 10-\mathrm{H} 10 \mathrm{~B}$ & 109.3 \\
\hline $\mathrm{C} 14-\mathrm{N} 4-\mathrm{C} 10$ & $115.6(7)$ & $\mathrm{H} 10 \mathrm{~A}-\mathrm{C} 10-\mathrm{H} 10 \mathrm{~B}$ & 108.0 \\
\hline $\mathrm{O} 2-\mathrm{C} 3-\mathrm{N} 2$ & $125.5(9)$ & $\mathrm{C} 12-\mathrm{C} 11-\mathrm{C} 10$ & $111.4(8)$ \\
\hline $\mathrm{O} 2-\mathrm{C} 3-\mathrm{C} 3 \mathrm{~A}$ & $126.0(8)$ & $\mathrm{C} 12-\mathrm{C} 11-\mathrm{H} 11 \mathrm{~A}$ & 109.4 \\
\hline $\mathrm{N} 2-\mathrm{C} 3-\mathrm{C} 3 \mathrm{~A}$ & $108.4(8)$ & $\mathrm{C} 10-\mathrm{C} 11-\mathrm{H} 11 \mathrm{~A}$ & 109.4 \\
\hline $\mathrm{C} 4-\mathrm{C} 3 \mathrm{~A}-\mathrm{C} 7 \mathrm{~A}$ & $121.2(9)$ & $\mathrm{C} 12-\mathrm{C} 11-\mathrm{H} 11 \mathrm{~B}$ & 109.4 \\
\hline $\mathrm{C} 4-\mathrm{C} 3 \mathrm{~A}-\mathrm{C} 3$ & $126.1(9)$ & $\mathrm{C} 10-\mathrm{C} 11-\mathrm{H} 11 \mathrm{~B}$ & 109.4 \\
\hline $\mathrm{C} 7 \mathrm{~A}-\mathrm{C} 3 \mathrm{~A}-\mathrm{C} 3$ & $112.7(8)$ & $\mathrm{H} 11 \mathrm{~A}-\mathrm{C} 11-\mathrm{H} 11 \mathrm{~B}$ & 108.0 \\
\hline $\mathrm{C} 3 \mathrm{~A}-\mathrm{C} 4-\mathrm{C} 5$ & $119.7(9)$ & $\mathrm{C} 11-\mathrm{C} 12-\mathrm{C} 13$ & $109.4(8)$ \\
\hline $\mathrm{C} 3 \mathrm{~A}-\mathrm{C} 4-\mathrm{H} 4$ & 120.2 & $\mathrm{C} 11-\mathrm{C} 12-\mathrm{H} 12 \mathrm{~A}$ & 109.8 \\
\hline $\mathrm{C} 5-\mathrm{C} 4-\mathrm{H} 4$ & 120.2 & $\mathrm{C} 13-\mathrm{C} 12-\mathrm{H} 12 \mathrm{~A}$ & 109.8 \\
\hline $\mathrm{C} 4-\mathrm{C} 5-\mathrm{C} 6$ & $120.8(9)$ & $\mathrm{C} 11-\mathrm{C} 12-\mathrm{H} 12 \mathrm{~B}$ & 109.8 \\
\hline $\mathrm{C} 4-\mathrm{C} 5-\mathrm{C} 8$ & $120.7(9)$ & $\mathrm{C} 13-\mathrm{C} 12-\mathrm{H} 12 \mathrm{~B}$ & 109.8 \\
\hline $\mathrm{C} 6-\mathrm{C} 5-\mathrm{C} 8$ & $118.5(9)$ & $\mathrm{H} 12 \mathrm{~A}-\mathrm{C} 12-\mathrm{H} 12 \mathrm{~B}$ & 108.2 \\
\hline $\mathrm{C} 7-\mathrm{C} 6-\mathrm{C} 5$ & $117.9(9)$ & $\mathrm{C} 14-\mathrm{C} 13-\mathrm{C} 12$ & $111.9(8)$ \\
\hline $\mathrm{C} 7-\mathrm{C} 6-\mathrm{H} 6$ & 121.0 & $\mathrm{C} 14-\mathrm{C} 13-\mathrm{H} 13 \mathrm{~A}$ & 109.2 \\
\hline $\mathrm{C} 5-\mathrm{C} 6-\mathrm{H} 6$ & 121.0 & $\mathrm{C} 12-\mathrm{C} 13-\mathrm{H} 13 \mathrm{~A}$ & 109.2 \\
\hline $\mathrm{C} 6-\mathrm{C} 7-\mathrm{C} 7 \mathrm{~A}$ & $122.1(9)$ & $\mathrm{C} 14-\mathrm{C} 13-\mathrm{H} 13 \mathrm{~B}$ & 109.2 \\
\hline $\mathrm{C} 6-\mathrm{C} 7-\mathrm{N} 3$ & $118.2(8)$ & $\mathrm{C} 12-\mathrm{C} 13-\mathrm{H} 13 \mathrm{~B}$ & 109.2 \\
\hline $\mathrm{C} 7 \mathrm{~A}-\mathrm{C} 7-\mathrm{N} 3$ & $119.7(8)$ & $\mathrm{H} 13 \mathrm{~A}-\mathrm{C} 13-\mathrm{H} 13 \mathrm{~B}$ & 107.9 \\
\hline $\mathrm{C} 3 \mathrm{~A}-\mathrm{C} 7 \mathrm{~A}-\mathrm{C} 7$ & $118.2(9)$ & $\mathrm{N} 4-\mathrm{C} 14-\mathrm{C} 13$ & $110.5(8)$ \\
\hline $\mathrm{C} 3 \mathrm{~A}-\mathrm{C} 7 \mathrm{~A}-\mathrm{S} 1$ & $113.2(7)$ & $\mathrm{N} 4-\mathrm{C} 14-\mathrm{H} 14 \mathrm{~A}$ & 109.5 \\
\hline $\mathrm{C} 7-\mathrm{C} 7 \mathrm{~A}-\mathrm{S} 1$ & $128.6(7)$ & $\mathrm{C} 13-\mathrm{C} 14-\mathrm{H} 14 \mathrm{~A}$ & 109.5 \\
\hline $\mathrm{F} 3-\mathrm{C} 8-\mathrm{F} 1$ & $106.7(8)$ & $\mathrm{N} 4-\mathrm{C} 14-\mathrm{H} 14 \mathrm{~B}$ & 109.5 \\
\hline $\mathrm{F} 3-\mathrm{C} 8-\mathrm{F} 2$ & $106.3(9)$ & $\mathrm{C} 13-\mathrm{C} 14-\mathrm{H} 14 \mathrm{~B}$ & 109.5 \\
\hline $\mathrm{F} 1-\mathrm{C} 8-\mathrm{F} 2$ & $105.8(8)$ & $\mathrm{H} 14 \mathrm{~A}-\mathrm{C} 14-\mathrm{H} 14 \mathrm{~B}$ & 108.1 \\
\hline $\mathrm{O} 1-\mathrm{S} 1-\mathrm{N} 2-\mathrm{C} 3$ & $121.9(6)$ & $\mathrm{N} 3-\mathrm{C} 7-\mathrm{C} 7 \mathrm{~A}-\mathrm{C} 3 \mathrm{~A}$ & $-175.3(8)$ \\
\hline
\end{tabular}




$\begin{array}{ll}\mathrm{C} 7 \mathrm{~A}-\mathrm{S} 1-\mathrm{N} 2-\mathrm{C} 3 & 14.1(6) \\ \mathrm{O} 1-\mathrm{S} 1-\mathrm{N} 2-\mathrm{C} 9 & -80.9(6) \\ \mathrm{C} 7 \mathrm{~A}-\mathrm{S} 1-\mathrm{N} 2-\mathrm{C} 9 & 171.3(6) \\ \mathrm{C} 9-\mathrm{N} 2-\mathrm{C} 3-\mathrm{O} 2 & 162.1(7) \\ \mathrm{S} 1-\mathrm{N} 2-\mathrm{C} 3-\mathrm{O} 2 & -171.7(8) \\ \mathrm{C} 9-\mathrm{N} 2-\mathrm{C} 3-\mathrm{C} 3 \mathrm{~A} & -17.1(9) \\ \mathrm{S} 1-\mathrm{N} 2-\mathrm{C} 3-\mathrm{C} 3 \mathrm{~A} & 10.7(14) \\ \mathrm{O} 2-\mathrm{C} 3-\mathrm{C} 3 \mathrm{~A}-\mathrm{C} 4 & -170.1(8) \\ \mathrm{N} 2-\mathrm{C} 3-\mathrm{C} 3 \mathrm{~A}-\mathrm{C} 4 & -168.2(9) \\ \mathrm{O} 2-\mathrm{C} 3-\mathrm{C} 3 \mathrm{~A}-\mathrm{C} 7 \mathrm{~A} & 11.0(10) \\ \mathrm{N} 2-\mathrm{C} 3-\mathrm{C} 3 \mathrm{~A}-\mathrm{C} 7 \mathrm{~A} & -2.8(13) \\ \mathrm{C} 7 \mathrm{~A}-\mathrm{C} 3 \mathrm{~A}-\mathrm{C} 4-\mathrm{C} 5 & 178.3(8) \\ \mathrm{C} 3-\mathrm{C} 3 \mathrm{~A}-\mathrm{C} 4-\mathrm{C} 5 & 3.8(13) \\ \mathrm{C} 3 \mathrm{~A}-\mathrm{C} 4-\mathrm{C} 5-\mathrm{C} 6 & -177.7(9) \\ \mathrm{C} 3 \mathrm{~A}-\mathrm{C} 4-\mathrm{C} 5-\mathrm{C} 8 & -1.2(13) \\ \mathrm{C} 4-\mathrm{C} 5-\mathrm{C} 6-\mathrm{C} 7 & -179.7(8) \\ \mathrm{C} 8-\mathrm{C} 5-\mathrm{C} 6-\mathrm{C} 7 & -2.5(13) \\ \mathrm{C} 5-\mathrm{C} 6-\mathrm{C} 7-\mathrm{C} 7 \mathrm{~A} & 176.4(8) \\ \mathrm{C} 5-\mathrm{C} 6-\mathrm{C} 7-\mathrm{N} 3 & -167.9(8) \\ \mathrm{O} 3-\mathrm{N} 3-\mathrm{C} 7-\mathrm{C} 6 & 13.0(12) \\ \mathrm{O} 4-\mathrm{N} 3-\mathrm{C} 7-\mathrm{C} 6 & 10.9(13) \\ \mathrm{O} 3-\mathrm{N} 3-\mathrm{C} 7-\mathrm{C} 7 \mathrm{~A} & -168.1(8) \\ \mathrm{O} 4-\mathrm{N} 3-\mathrm{C} 7-\mathrm{C} 7 \mathrm{~A} & -0.8(13) \\ \mathrm{C} 4-\mathrm{C} 3 \mathrm{~A}-\mathrm{C} 7 \mathrm{~A}-\mathrm{C} 7 & 178.2(8) \\ \mathrm{C} 3-\mathrm{C} 3 \mathrm{~A}-\mathrm{C} 7 \mathrm{~A}-\mathrm{C} 7 & 180.0(7) \\ \mathrm{C} 4-\mathrm{C} 3 \mathrm{~A}-\mathrm{C} 7 \mathrm{~A}-\mathrm{S} 1 & -1.1(10) \\ \mathrm{C} 3-\mathrm{C} 3 \mathrm{~A}-\mathrm{C} 7 \mathrm{~A}-\mathrm{S} 1 & 3.5(13) \\ \mathrm{C} 6-\mathrm{C} 7-\mathrm{C} 7 \mathrm{~A}-\mathrm{C} 3 \mathrm{~A} & \end{array}$

$\begin{array}{ll}\mathrm{C} 6-\mathrm{C} 7-\mathrm{C} 7 \mathrm{~A}-\mathrm{S} 1 & -177.4(7) \\ \mathrm{N} 3-\mathrm{C} 7-\mathrm{C} 7 \mathrm{~A}-\mathrm{S} 1 & 3.8(13) \\ \mathrm{O} 1-\mathrm{S} 1-\mathrm{C} 7 \mathrm{~A}-\mathrm{C} 3 \mathrm{~A} & -114.4(7) \\ \mathrm{N} 2-\mathrm{S} 1-\mathrm{C} 7 \mathrm{~A}-\mathrm{C} 3 \mathrm{~A} & -6.9(7) \\ \mathrm{O} 1-\mathrm{S} 1-\mathrm{C} 7 \mathrm{~A}-\mathrm{C} 7 & 66.4(9) \\ \mathrm{N} 2-\mathrm{S} 1-\mathrm{C} 7 \mathrm{~A}-\mathrm{C} 7 & 173.9(9) \\ \mathrm{C} 4-\mathrm{C} 5-\mathrm{C} 8-\mathrm{F} 3 & 20.3(13) \\ \mathrm{C} 6-\mathrm{C} 5-\mathrm{C} 8-\mathrm{F} 3 & -161.2(8) \\ \mathrm{C} 4-\mathrm{C} 5-\mathrm{C} 8-\mathrm{F} 1 & -100.2(11) \\ \mathrm{C} 6-\mathrm{C} 5-\mathrm{C} 8-\mathrm{F} 1 & 78.4(11) \\ \mathrm{C} 4-\mathrm{C} 5-\mathrm{C} 8-\mathrm{F} 2 & 140.5(9) \\ \mathrm{C} 6-\mathrm{C} 5-\mathrm{C} 8-\mathrm{F} 2 & -41.0(13) \\ \mathrm{C} 14-\mathrm{N} 4-\mathrm{C} 9-\mathrm{O} 5 & 165.6(8) \\ \mathrm{C} 10-\mathrm{N} 4-\mathrm{C} 9-\mathrm{O} 5 & -12.6(13) \\ \mathrm{C} 14-\mathrm{N} 4-\mathrm{C} 9-\mathrm{N} 2 & -13.8(13) \\ \mathrm{C} 10-\mathrm{N} 4-\mathrm{C} 9-\mathrm{N} 2 & 168.1(7) \\ \mathrm{C} 3-\mathrm{N} 2-\mathrm{C} 9-\mathrm{O} 5 & 128.9(9) \\ \mathrm{S} 1-\mathrm{N} 2-\mathrm{C} 9-\mathrm{O} 5 & -26.2(10) \\ \mathrm{C} 3-\mathrm{N} 2-\mathrm{C} 9-\mathrm{N} 4 & -51.7(12) \\ \mathrm{S} 1-\mathrm{N} 2-\mathrm{C} 9-\mathrm{N} 4 & 153.3(7) \\ \mathrm{C} 9-\mathrm{N} 4-\mathrm{C} 10-\mathrm{C} 11 & 126.6(9) \\ \mathrm{C} 14-\mathrm{N} 4-\mathrm{C} 10-\mathrm{C} 11 & -51.8(11) \\ \mathrm{N} 4-\mathrm{C} 10-\mathrm{C} 11-\mathrm{C} 12 & 52.2(11) \\ \mathrm{C} 10-\mathrm{C} 11-\mathrm{C} 12-\mathrm{C} 13 & -54.9(11) \\ \mathrm{C} 11-\mathrm{C} 12-\mathrm{C} 13-\mathrm{C} 14 & 57.0(11) \\ \mathrm{C} 9-\mathrm{N} 4-\mathrm{C} 14-\mathrm{C} 13 & -125.1(10) \\ \mathrm{C} 10-\mathrm{N} 4-\mathrm{C} 14-\mathrm{C} 13 & 53.0(10) \\ \mathrm{C} 12-\mathrm{C} 13-\mathrm{C} 14-\mathrm{N} 4 & -54.9(10)\end{array}$

2-[(S)-2-Methyl-1,4-dioxa-8-azaspiro[4.5]decane-8-carbonyl]-7-

nitro-5-(trifluoromethyl)benzo[d] isothiazol-3(2H)-one 1-oxide (4)

Crystal data

$\mathrm{C}_{17} \mathrm{H}_{16} \mathrm{~F}_{3} \mathrm{~N}_{3} \mathrm{O}_{7} \mathrm{~S}$

$M_{r}=463.39$

Monoclinic, $P 2_{1}$

$a=8.8165(2) \AA$

$b=16.1649$ (4) $\AA$

$c=13.4246(3) \AA$

$\beta=90.0022(12)^{\circ}$

$V=1913.24(8) \AA^{3}$

$Z=4$

\section{Data collection}

Bruker PHOTON-II

diffractometer

Radiation source: Incoatec micro-focus Detector resolution: 7.41 pixels $\mathrm{mm}^{-1}$ $\varphi$ - and $\omega$-scans
$F(000)=952$

$D_{\mathrm{x}}=1.609 \mathrm{Mg} \mathrm{m}^{-3}$

$\mathrm{Cu} K \alpha$ radiation, $\lambda=1.54178 \AA$

Cell parameters from 9779 reflections

$\theta=3.3-70.3^{\circ}$

$\mu=2.23 \mathrm{~mm}^{-1}$

$T=120 \mathrm{~K}$

Blade, colorless

$0.25 \times 0.07 \times 0.04 \mathrm{~mm}$ 


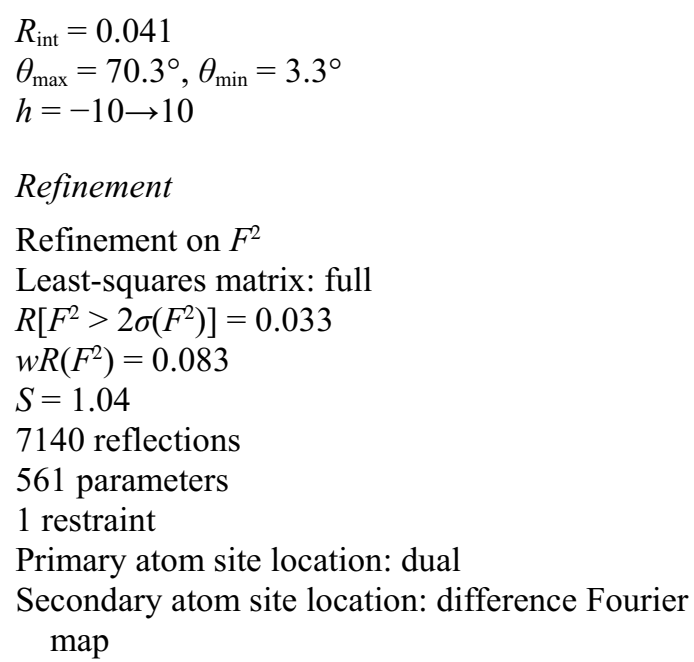

$R_{\text {int }}=0.041$

$\theta_{\max }=70.3^{\circ}, \theta_{\min }=3.3^{\circ}$

Refinement

Refinement on $F^{2}$

Least-squares matrix: full

$R\left[F^{2}>2 \sigma\left(F^{2}\right)\right]=0.033$

$S=1.04$

7140 reflections

561 parameters

1 restraint

Secondary atom site location: difference Fourier map

$k=-19 \rightarrow 19$

$l=-16 \rightarrow 16$

Hydrogen site location: inferred from neighbouring sites

$\mathrm{H}$-atom parameters constrained

$w=1 /\left[\sigma^{2}\left(F_{\mathrm{o}}^{2}\right)+(0.0446 P)^{2}+0.5798 P\right]$

where $P=\left(F_{\mathrm{o}}{ }^{2}+2 F_{\mathrm{c}}{ }^{2}\right) / 3$

$(\Delta / \sigma)_{\max }<0.001$

$\Delta \rho_{\max }=0.61 \mathrm{e}^{-3}$

$\Delta \rho_{\min }=-0.31$ e $\AA^{-3}$

Absolute structure: Flack $x$ determined using 3080 quotients $[(\mathrm{I}+)-(\mathrm{I}-)] /[(\mathrm{I}+)+(\mathrm{I}-)]$ (Parsons et al., 2013)

Absolute structure parameter: 0.017 (6)

Special details

Geometry. All esds (except the esd in the dihedral angle between two 1.s. planes) are estimated using the full covariance matrix. The cell esds are taken into account individually in the estimation of esds in distances, angles and torsion angles; correlations between esds in cell parameters are only used when they are defined by crystal symmetry. An approximate (isotropic) treatment of cell esds is used for estimating esds involving 1.s. planes.

Refinement. Although the monoclinic beta angle is close to $90^{\circ}$, the structure is monoclinic with no sign of twinning. Averaging the diffraction data under mmm Laue symmetry results in $\mathrm{R}(\mathrm{sym})=0.305$ (XPREP).

Fractional atomic coordinates and isotropic or equivalent isotropic displacement parameters $\left(\AA^{2}\right)$

\begin{tabular}{lllll}
\hline & $x$ & $y$ & $z$ & $U_{\text {iso }} * U_{\text {eq }}$ \\
\hline S1_1 & $0.30602(8)$ & $0.66421(5)$ & $0.53028(5)$ & $0.02508(16)$ \\
F1_1 & $0.4878(3)$ & $0.5408(2)$ & $0.0698(2)$ & $0.0671(9)$ \\
F2_1 & $0.3554(4)$ & $0.43821(16)$ & $0.11563(19)$ & $0.0611(8)$ \\
F3_1 & $0.2495(4)$ & $0.5428(2)$ & $0.05546(19)$ & $0.0698(9)$ \\
O1_1 & $0.1439(2)$ & $0.66606(16)$ & $0.55526(16)$ & $0.0311(5)$ \\
O2_1 & $0.4782(3)$ & $0.44663(16)$ & $0.50398(18)$ & $0.0351(6)$ \\
O3_1 & $0.2457(4)$ & $0.79269(17)$ & $0.4081(2)$ & $0.0440(6)$ \\
O4_1 & $0.1989(3)$ & $0.79614(17)$ & $0.2497(2)$ & $0.0411(6)$ \\
O5_1 & $0.4618(3)$ & $0.62275(15)$ & $0.70901(17)$ & $0.0303(5)$ \\
O6_1 & $0.2306(3)$ & $0.33735(16)$ & $0.8664(2)$ & $0.0355(6)$ \\
O7_1 & $0.4331(3)$ & $0.25355(16)$ & $0.8326(2)$ & $0.0372(6)$ \\
N2_1 & $0.3682(3)$ & $0.56598(17)$ & $0.5661(2)$ & $0.0257(6)$ \\
N3_1 & $0.2405(3)$ & $0.76066(18)$ & $0.3254(2)$ & $0.0321(6)$ \\
N4_1 & $0.3743(3)$ & $0.49099(18)$ & $0.7162(2)$ & $0.0272(6)$ \\
C3_1 & $0.4135(4)$ & $0.5114(2)$ & $0.4911(2)$ & $0.0270(7)$ \\
C3A_1 & $0.3715(4)$ & $0.5490(2)$ & $0.3938(2)$ & $0.0251(6)$ \\
C4_1 & $0.3832(3)$ & $0.5100(2)$ & $0.3017(2)$ & $0.0251(6)$ \\
H4_1 & 0.413697 & 0.453809 & 0.296739 & $0.030^{*}$ \\
C5_1 & $0.3484(3)$ & $0.5563(2)$ & $0.2168(2)$ & $0.0256(6)$ \\
C6_1 & $0.3028(4)$ & $0.6385(2)$ & $0.2245(2)$ & $0.0278(7)$ \\
H6_1 & 0.280891 & 0.669736 & 0.166306 & $0.033 *$ \\
C7_1 & $0.2897(3)$ & $0.6742(2)$ & $0.3177(2)$ & $0.0261(6)$ \\
& & & &
\end{tabular}




\begin{tabular}{|c|c|c|c|c|}
\hline C7A_1 & $0.3224(3)$ & $0.6294(2)$ & $0.4026(2)$ & $0.0243(6)$ \\
\hline C8_1 & $0.3641(4)$ & $0.5181(2)$ & $0.1151(2)$ & $0.0300(7)$ \\
\hline C9_1 & $0.4068(4)$ & $0.5614(2)$ & $0.6702(2)$ & $0.0262(6)$ \\
\hline C10_1 & $0.4126(4)$ & $0.4841(2)$ & $0.8225(2)$ & $0.0300(7)$ \\
\hline H10A_1 & 0.320432 & 0.493256 & 0.863078 & $0.036^{*}$ \\
\hline H10B_1 & 0.487716 & 0.527145 & 0.840268 & $0.036^{*}$ \\
\hline C11_1 & $0.4776(4)$ & $0.3993(2)$ & $0.8452(3)$ & $0.0319(7)$ \\
\hline H11A_1 & 0.576074 & 0.392673 & 0.810696 & $0.038^{*}$ \\
\hline H11B_1 & 0.495561 & 0.394122 & 0.917723 & $0.038^{*}$ \\
\hline C12_1 & $0.3696(4)$ & $0.3320(2)$ & $0.8115(3)$ & $0.0305(7)$ \\
\hline C13_1 & $0.3324(4)$ & $0.3413(2)$ & $0.7007(3)$ & $0.0318(7)$ \\
\hline H13A_1 & 0.425391 & 0.333099 & 0.660625 & $0.038^{*}$ \\
\hline H13B_1 & 0.257375 & 0.298890 & 0.680770 & $0.038^{*}$ \\
\hline C14_1 & $0.2686(4)$ & $0.4268(2)$ & $0.6816(3)$ & $0.0305(7)$ \\
\hline H14A_1 & 0.249873 & 0.433853 & 0.609366 & $0.037^{*}$ \\
\hline H14B_1 & 0.170498 & 0.432827 & 0.716744 & $0.037^{*}$ \\
\hline C15_1 & $0.2153(4)$ & $0.2645(2)$ & $0.9264(3)$ & $0.0357(8)$ \\
\hline H15_1 & 0.258964 & 0.274399 & 0.994133 & $0.043^{*}$ \\
\hline C16_1 & $0.3134(4)$ & $0.2035(2)$ & $0.8696(3)$ & $0.0387(8)$ \\
\hline H16A_1 & 0.352563 & 0.159634 & 0.914125 & $0.046^{*}$ \\
\hline H16B_1 & 0.255997 & 0.177567 & 0.814418 & $0.046^{*}$ \\
\hline C17_1 & $0.0503(5)$ & $0.2413(3)$ & 0.9349 (3) & 0.0409 (9) \\
\hline H17A_1 & -0.003992 & 0.284440 & 0.971850 & $0.061^{*}$ \\
\hline H17B_1 & 0.041028 & 0.188541 & 0.970358 & $0.061 *$ \\
\hline H17C_1 & 0.006466 & 0.235913 & 0.868172 & $0.061^{*}$ \\
\hline S1_2 & $0.80116(8)$ & $0.67422(5)$ & $0.49508(5)$ & $0.02466(16)$ \\
\hline F1_2 & $0.9905(3)$ & $0.52933(17)$ & $0.94230(18)$ & $0.0502(6)$ \\
\hline $\mathrm{F} 2 \_2$ & $0.8580(3)$ & $0.42752(15)$ & $0.89242(16)$ & $0.0505(6)$ \\
\hline F3_2 & $0.7516(3)$ & $0.5301(2)$ & $0.96310(18)$ & $0.0598(8)$ \\
\hline O1_2 & $0.6391(3)$ & $0.67522(16)$ & $0.46951(17)$ & $0.0314(5)$ \\
\hline O2_2 & $0.9930(3)$ & $0.46106(16)$ & $0.50856(17)$ & $0.0317(5)$ \\
\hline O3_2 & $0.7350(3)$ & $0.79653(17)$ & $0.6287(2)$ & $0.0416(6)$ \\
\hline O4_2 & $0.6666(3)$ & $0.78627(17)$ & $0.7835(2)$ & $0.0387(6)$ \\
\hline O5_2 & $0.9631(3)$ & $0.63962(15)$ & $0.31252(17)$ & $0.0312(5)$ \\
\hline O6_2 & $0.7628(3)$ & $0.34331(14)$ & $0.15282(17)$ & $0.0278(5)$ \\
\hline O7_2 & $0.9657(3)$ & $0.26758(15)$ & $0.19997(19)$ & $0.0310(5)$ \\
\hline N2_2 & $0.8690(3)$ & $0.57884(17)$ & $0.4538(2)$ & $0.0250(5)$ \\
\hline N3_2 & $0.7211(3)$ & $0.75800(18)$ & $0.7071(2)$ & $0.0299(6)$ \\
\hline N4_2 & $0.8767(3)$ & $0.50701(17)$ & $0.30231(19)$ & $0.0258(5)$ \\
\hline C3_2 & $0.9198(4)$ & $0.5226(2)$ & $0.5250(2)$ & $0.0253(6)$ \\
\hline C3A_2 & $0.8723(3)$ & $0.5537(2)$ & $0.6242(2)$ & $0.0237(6)$ \\
\hline C4_2 & $0.8831(3)$ & $0.5105(2)$ & $0.7132(2)$ & $0.0244(6)$ \\
\hline H4_2 & 0.917693 & 0.454873 & 0.714581 & $0.029^{*}$ \\
\hline C5_2 & $0.8417(4)$ & $0.5513(2)$ & $0.8004(2)$ & $0.0253(6)$ \\
\hline C6_2 & $0.7886(3)$ & $0.6322(2)$ & $0.7985(2)$ & $0.0256(6)$ \\
\hline H6_2 & 0.760764 & 0.659404 & 0.858474 & $0.031^{*}$ \\
\hline C7_2 & $0.7769(3)$ & $0.6725(2)$ & $0.7080(2)$ & $0.0256(6)$ \\
\hline C7A_2 & $0.8166(3)$ & $0.6337(2)$ & $0.6208(2)$ & $0.0243(6)$ \\
\hline
\end{tabular}




$\begin{array}{lllll}\text { C8_2 } & 0.8602(4) & 0.5086(2) & 0.8995(2) & 0.0278(7) \\ \text { C9_2 } & 0.9082(3) & 0.5775(2) & 0.3495(2) & 0.0247(6) \\ \text { C10_2 } & 0.9211(4) & 0.4984(2) & 0.1973(2) & 0.0286(7) \\ \text { H10C_2 } & 0.992716 & 0.543109 & 0.179110 & 0.034^{*} \\ \text { H10D_2 } & 0.830364 & 0.503397 & 0.154230 & 0.034^{*} \\ \text { C11_2 } & 0.9963(4) & 0.4141(2) & 0.1808(2) & 0.0296(7) \\ \text { H11C_2 } & 1.019612 & 0.407034 & 0.109177 & 0.036^{*} \\ \text { H11D_2 } & 1.092943 & 0.411788 & 0.218231 & 0.036^{*} \\ \text { C12_2 } & 0.8936(4) & 0.3445(2) & 0.2150(2) & 0.0267(7) \\ \text { C13_2 } & 0.8496(4) & 0.3570(2) & 0.3241(2) & 0.0279(7) \\ \text { H13C_2 } & 0.778394 & 0.312906 & 0.344953 & 0.033^{*} \\ \text { H13D_2 } & 0.941200 & 0.353360 & 0.366559 & 0.033^{*} \\ \text { C14_2 } & 0.7756(4) & 0.4407(2) & 0.3373(2) & 0.0277(7) \\ \text { H14C_2 } & 0.679456 & 0.442399 & 0.299286 & 0.033^{*} \\ \text { H14D_2 } & 0.751547 & 0.449476 & 0.408546 & 0.033^{*} \\ \text { C15_2 } & 0.7050(4) & 0.2603(2) & 0.1594(3) & 0.0294(7) \\ \text { H15B_2 } & 0.632899 & 0.255825 & 0.216599 & 0.035^{*} \\ \text { C16_2 } & 0.8482(4) & 0.2086(2) & 0.1806(3) & 0.0307(7) \\ \text { H16C_2 } & 0.832426 & 0.172305 & 0.239069 & 0.037^{*} \\ \text { H16D_2 } & 0.874416 & 0.173854 & 0.122391 & 0.037^{*} \\ \text { C17_2 } & 0.6243(5) & 0.2395(3) & 0.0633(3) & 0.0399(9) \\ \text { H17D_2 } & 0.535456 & 0.275423 & 0.055739 & 0.060^{*} \\ \text { H17E_2 } & 0.591606 & 0.181582 & 0.064828 & 0.060^{*} \\ \text { H17F_2 } & 0.693369 & 0.248058 & 0.007064 & 0.060^{*}\end{array}$

Atomic displacement parameters $\left(\AA^{2}\right)$

\begin{tabular}{lllllll}
\hline & $U^{11}$ & $U^{22}$ & $U^{33}$ & $U^{12}$ & $U^{13}$ & $U^{23}$ \\
\hline S1_1 & $0.0265(4)$ & $0.0257(4)$ & $0.0231(3)$ & $-0.0001(3)$ & $0.0001(2)$ & $-0.0018(3)$ \\
F1_1 & $0.0607(17)$ & $0.085(2)$ & $0.0554(16)$ & $-0.0381(15)$ & $0.0340(13)$ & $-0.0312(15)$ \\
F2_1 & $0.108(2)$ & $0.0411(14)$ & $0.0342(12)$ & $-0.0178(14)$ & $0.0175(13)$ & $-0.0051(10)$ \\
F3_1 & $0.0717(19)$ & $0.104(2)$ & $0.0339(13)$ & $0.0250(17)$ & $-0.0134(12)$ & $-0.0137(14)$ \\
O1_1 & $0.0277(11)$ & $0.0361(12)$ & $0.0295(11)$ & $0.0008(10)$ & $0.0046(8)$ & $-0.0032(11)$ \\
O2_1 & $0.0442(15)$ & $0.0354(14)$ & $0.0256(12)$ & $0.0118(11)$ & $0.0011(10)$ & $0.0025(10)$ \\
O3_1 & $0.0616(18)$ & $0.0320(13)$ & $0.0383(15)$ & $0.0057(12)$ & $-0.0041(12)$ & $-0.0014(11)$ \\
O4_1 & $0.0498(16)$ & $0.0341(13)$ & $0.0394(15)$ & $0.0042(12)$ & $-0.0013(12)$ & $0.0121(12)$ \\
O5_1 & $0.0313(12)$ & $0.0327(13)$ & $0.0269(12)$ & $-0.0043(10)$ & $-0.0019(9)$ & $-0.0024(10)$ \\
O6_1 & $0.0355(13)$ & $0.0308(12)$ & $0.0403(14)$ & $0.0049(10)$ & $0.0104(10)$ & $0.0064(10)$ \\
O7_1 & $0.0356(14)$ & $0.0310(13)$ & $0.0450(15)$ & $0.0076(10)$ & $-0.0017(11)$ & $0.0009(11)$ \\
N2_1 & $0.0293(14)$ & $0.0266(14)$ & $0.0213(13)$ & $0.0028(10)$ & $-0.0002(10)$ & $0.0006(10)$ \\
N3_1 & $0.0345(16)$ & $0.0279(14)$ & $0.0339(16)$ & $0.0000(12)$ & $0.0023(12)$ & $0.0070(13)$ \\
N4_1 & $0.0302(14)$ & $0.0314(15)$ & $0.0201(13)$ & $-0.0038(11)$ & $-0.0022(10)$ & $-0.0010(11)$ \\
C3_1 & $0.0274(16)$ & $0.0316(18)$ & $0.0221(15)$ & $0.0019(13)$ & $0.0007(11)$ & $0.0020(13)$ \\
C3A_1 & $0.0225(15)$ & $0.0281(16)$ & $0.0247(15)$ & $-0.0007(12)$ & $0.0001(11)$ & $0.0044(13)$ \\
C4_1 & $0.0218(14)$ & $0.0280(16)$ & $0.0255(16)$ & $-0.0001(12)$ & $0.0028(11)$ & $-0.0004(12)$ \\
C5_1 & $0.0209(15)$ & $0.0323(16)$ & $0.0235(15)$ & $-0.0057(12)$ & $0.0026(11)$ & $0.0022(13)$ \\
C6_1 & $0.0232(15)$ & $0.0346(17)$ & $0.0255(16)$ & $-0.0042(13)$ & $0.0011(11)$ & $0.0056(13)$ \\
C7_1 & $0.0231(14)$ & $0.0262(15)$ & $0.0290(15)$ & $-0.0041(13)$ & $0.0009(11)$ & $0.0010(14)$
\end{tabular}




\begin{tabular}{|c|c|c|c|c|c|c|}
\hline C7A_1 & $0.0205(14)$ & $0.0282(16)$ & $0.0242(15)$ & $-0.0038(12)$ & $0.0018(11)$ & $0.0006(12)$ \\
\hline C8_1 & $0.0343(18)$ & $0.0325(18)$ & $0.0231(16)$ & $-0.0071(14)$ & $0.0047(12)$ & $0.0041(13)$ \\
\hline C9_1 & $0.0215(15)$ & $0.0340(17)$ & $0.0232(15)$ & $0.0024(12)$ & $0.0010(11)$ & $-0.0023(13)$ \\
\hline C10_1 & $0.0325(18)$ & $0.0358(19)$ & $0.0216(15)$ & $-0.0007(14)$ & $-0.0034(12)$ & $-0.0015(13)$ \\
\hline C11_1 & $0.0322(18)$ & $0.0375(19)$ & $0.0259(16)$ & $0.0016(14)$ & $-0.0043(13)$ & $0.0000(14)$ \\
\hline C12_1 & $0.0294(17)$ & $0.0330(17)$ & $0.0292(17)$ & $0.0024(14)$ & $0.0025(13)$ & $-0.0007(13)$ \\
\hline C13_1 & $0.0313(17)$ & $0.0339(18)$ & $0.0302(17)$ & $-0.0019(14)$ & $-0.0029(13)$ & $-0.0062(14)$ \\
\hline C14_1 & $0.0269(17)$ & $0.0356(18)$ & $0.0289(16)$ & $-0.0039(14)$ & $-0.0046(13)$ & $-0.0003(14)$ \\
\hline C15_1 & $0.040(2)$ & $0.0369(19)$ & $0.0306(18)$ & $-0.0008(15)$ & $-0.0060(14)$ & $0.0088(15)$ \\
\hline C16_1 & $0.038(2)$ & $0.0299(17)$ & $0.048(2)$ & $0.0023(14)$ & $-0.0051(16)$ & $0.0084(16)$ \\
\hline C17_1 & $0.042(2)$ & $0.040(2)$ & $0.041(2)$ & $-0.0031(16)$ & $0.0030(16)$ & $0.0087(16)$ \\
\hline S1_2 & $0.0262(4)$ & $0.0249(4)$ & $0.0229(3)$ & $0.0013(3)$ & $-0.0001(2)$ & $0.0016(3)$ \\
\hline F1_2 & $0.0472(13)$ & $0.0622(15)$ & $0.0411(13)$ & $-0.0194(11)$ & $-0.0207(10)$ & $0.0156(11)$ \\
\hline F2_2 & $0.089(2)$ & $0.0348(12)$ & $0.0277(11)$ & $-0.0093(12)$ & -0.0064 (11) & $0.0037(9)$ \\
\hline F3_2 & $0.0599(16)$ & $0.090(2)$ & $0.0293(12)$ & $0.0263(15)$ & $0.0181(11)$ & $0.0136(12)$ \\
\hline O1_2 & $0.0281(11)$ & $0.0358(12)$ & $0.0303(11)$ & $0.0032(10)$ & $-0.0041(8)$ & $0.0019(11)$ \\
\hline $\mathrm{O} 2 \_2$ & $0.0371(13)$ & $0.0344(13)$ & $0.0236(11)$ & $0.0105(10)$ & $-0.0014(9)$ & $-0.0023(9)$ \\
\hline O3_2 & $0.0545(17)$ & $0.0306(13)$ & $0.0398(15)$ & $0.0085(12)$ & $-0.0005(12)$ & 0.0009 (12) \\
\hline O4_2 & $0.0359(14)$ & $0.0376(14)$ & $0.0425(15)$ & $0.0036(11)$ & $0.0047(11)$ & $-0.0128(11)$ \\
\hline O5_2 & $0.0359(13)$ & $0.0322(12)$ & $0.0253(11)$ & $-0.0054(10)$ & $0.0045(9)$ & $0.0018(9)$ \\
\hline O6_2 & $0.0274(12)$ & $0.0309(12)$ & $0.0251(11)$ & $0.0000(9)$ & $-0.0029(9)$ & $0.0013(9)$ \\
\hline O7_2 & $0.0259(12)$ & $0.0286(12)$ & $0.0384(13)$ & $0.0027(9)$ & $-0.0011(9)$ & $-0.0020(10)$ \\
\hline N2_2 & $0.0286(13)$ & $0.0263(14)$ & $0.0202(13)$ & $0.0015(11)$ & $0.0008(10)$ & $-0.0010(10)$ \\
\hline N3_2 & $0.0282(15)$ & $0.0272(14)$ & $0.0343(16)$ & $0.0006(11)$ & $-0.0024(11)$ & $-0.0077(12)$ \\
\hline N4_2 & $0.0267(13)$ & $0.0299(14)$ & $0.0206(13)$ & $-0.0018(11)$ & $0.0033(10)$ & $0.0014(11)$ \\
\hline C3_2 & $0.0275(16)$ & $0.0271(16)$ & $0.0214(14)$ & $0.0008(13)$ & $-0.0006(11)$ & $-0.0017(12)$ \\
\hline C3A_2 & $0.0215(15)$ & $0.0283(16)$ & $0.0213(15)$ & $0.0002(12)$ & $-0.0001(11)$ & $-0.0027(12)$ \\
\hline C4_2 & $0.0216(14)$ & $0.0282(16)$ & $0.0236(15)$ & $0.0007(12)$ & $0.0000(11)$ & $-0.0006(12)$ \\
\hline C5_2 & $0.0215(15)$ & $0.0336(17)$ & $0.0206(15)$ & $-0.0049(12)$ & $-0.0001(11)$ & $-0.0006(13)$ \\
\hline C6_2 & $0.0214(14)$ & $0.0310(16)$ & $0.0244(15)$ & $-0.0016(12)$ & $0.0010(11)$ & $-0.0054(13)$ \\
\hline C7_2 & $0.0194(13)$ & $0.0268(15)$ & $0.0306(15)$ & $-0.0011(13)$ & $0.0000(11)$ & $-0.0050(14)$ \\
\hline C7A_2 & $0.0209(14)$ & $0.0269(15)$ & $0.0251(15)$ & $-0.0030(12)$ & $-0.0011(11)$ & $0.0015(12)$ \\
\hline C8_2 & $0.0274(16)$ & $0.0344(18)$ & $0.0215(15)$ & $-0.0023(13)$ & $0.0004(12)$ & $-0.0017(13)$ \\
\hline C9_2 & $0.0219(14)$ & $0.0323(17)$ & $0.0199(14)$ & $0.0025(12)$ & $-0.0001(11)$ & $0.0027(12)$ \\
\hline C10_2 & $0.0337(17)$ & $0.0311(17)$ & $0.0211(15)$ & $-0.0033(13)$ & $0.0031(12)$ & $0.0023(13)$ \\
\hline C11_2 & $0.0291(17)$ & $0.0358(18)$ & $0.0239(15)$ & $-0.0030(13)$ & $0.0039(12)$ & $-0.0022(13)$ \\
\hline C12_2 & $0.0239(15)$ & $0.0312(17)$ & $0.0251(16)$ & $0.0036(13)$ & $-0.0009(12)$ & $0.0012(13)$ \\
\hline C13_2 & $0.0308(17)$ & $0.0297(17)$ & $0.0230(15)$ & $-0.0026(13)$ & $-0.0002(12)$ & $0.0017(13)$ \\
\hline C14_2 & $0.0280(16)$ & $0.0330(17)$ & $0.0221(15)$ & $-0.0048(13)$ & $0.0051(12)$ & $0.0015(13)$ \\
\hline C15_2 & $0.0274(17)$ & $0.0319(17)$ & $0.0288(16)$ & $0.0000(13)$ & $-0.0001(13)$ & $-0.0015(13)$ \\
\hline C16_2 & $0.0312(18)$ & $0.0289(16)$ & $0.0321(17)$ & $-0.0002(14)$ & $0.0017(13)$ & $-0.0020(14)$ \\
\hline C17_2 & $0.041(2)$ & $0.039(2)$ & $0.039(2)$ & $-0.0019(16)$ & $-0.0103(16)$ & $-0.0053(16)$ \\
\hline
\end{tabular}

Geometric parameters $\left(A,{ }^{o}\right)$

\begin{tabular}{llll}
\hline $\mathrm{S} 1 \_1-\mathrm{O} 1 \_1$ & $1.468(2)$ & $\mathrm{S} 1 \_2-\mathrm{O} 1 \_2$ & $1.470(2)$ \\
$\mathrm{S} 1 \_1-\mathrm{N} 2 \_1$ & $1.747(3)$ & $\mathrm{S} 1 \_2-\mathrm{N} 2 \_2$ & $1.744(3)$ \\
$\mathrm{S} 1 \_1-\mathrm{C} 7 \mathrm{~A} \_1$ & $1.810(3)$ & $\mathrm{S} 1 \_2-\mathrm{C} 7 \mathrm{~A} \_2$ & $1.815(3)$
\end{tabular}




\begin{tabular}{|c|c|c|c|}
\hline $\mathrm{F} 1 \_1-\mathrm{C} 8 \_1$ & $1.302(4)$ & F1_2-C8_2 & $1.327(4)$ \\
\hline $\mathrm{F} 2 \_1-\mathrm{C} 8 \_1$ & $1.293(5)$ & $\mathrm{F} 2 \_2-\mathrm{C} 8 \_2$ & $1.315(4)$ \\
\hline $\mathrm{F} 3{ }_{-}^{-} 1-\mathrm{C} 8{ }_{-}^{-} 1$ & $1.349(5)$ & $\mathrm{F} 3 \_2-\mathrm{C} 8 \_2$ & $1.329(4)$ \\
\hline $\mathrm{O} 2 \_1-\mathrm{C} 3 \_1$ & $1.204(4)$ & $\mathrm{O} 2 \_2-\mathrm{C} 3 \_2$ & $1.206(4)$ \\
\hline $\mathrm{O} 3 \_1-\mathrm{N} 3 \_1$ & $1.226(4)$ & $\mathrm{O} 3 \_2-\mathrm{N} 3 \_2$ & $1.229(4)$ \\
\hline $\mathrm{O} 4 \_1-\mathrm{N} 3 \_1$ & $1.223(4)$ & $\mathrm{O} 4 \_2-\mathrm{N} 3 \_2$ & $1.221(4)$ \\
\hline $\mathrm{O} 5 \_1-\mathrm{C} 9 \_1$ & $1.221(4)$ & O5_2-C9_2 & $1.220(4)$ \\
\hline O6_1-C12_1 & $1.432(4)$ & O6_2- $\mathrm{C} 12 \_2$ & $1.424(4)$ \\
\hline O6_1-C15_1 & $1.434(4)$ & O6_2-C15_2 & $1.438(4)$ \\
\hline $\mathrm{O} 7 \_1-\mathrm{C} 12 \_1$ & $1.415(4)$ & O7_2-C12_2 & $1.411(4)$ \\
\hline O7_1-C16_1 & $1.419(5)$ & O7_2-C16_2 & $1.431(4)$ \\
\hline $\mathrm{N} 2 \_1-\mathrm{C} 3 \_1$ & $1.397(4)$ & $\mathrm{N} 2 \_2-\mathrm{C} 3 \_2$ & $1.393(4)$ \\
\hline $\mathrm{N} 2 \_1-\mathrm{C} 9 \_1$ & $1.440(4)$ & N2_2-C9_2 & $1.442(4)$ \\
\hline N3_1-C7_1 & $1.466(5)$ & N3_2-C7_2 & $1.466(5)$ \\
\hline N4_1-C9_1 & $1.326(5)$ & N4_2-C9_2 & $1.333(4)$ \\
\hline N4_1-C10_1 & $1.470(4)$ & N4_2-C10_2 & $1.470(4)$ \\
\hline N4_1-C14_1 & $1.470(4)$ & N4_2-C14_2 & $1.471(4)$ \\
\hline C3_1-C3A_1 & $1.488(4)$ & C3_2-C3A_2 & $1.484(4)$ \\
\hline C3A_1-C7A_1 & $1.375(5)$ & C3A_2-C7A_2 & $1.384(5)$ \\
\hline C3A_1-C4_1 & $1.391(5)$ & C3A_2-C4_2 & $1.386(5)$ \\
\hline C4_1-C5_1 & $1.398(4)$ & C4_2-C5_2 & $1.392(4)$ \\
\hline $\mathrm{C} 4 \_1-\mathrm{H} 4 \_1$ & 0.9500 & $\mathrm{C} 4 \_2-\mathrm{H} 4 \_2$ & 0.9500 \\
\hline C5_1-C6_1 & $1.392(5)$ & C5_2-C6_2 & $1.389(5)$ \\
\hline C5 $11-\mathrm{C} 8 \_1$ & $1.505(5)$ & C5 $52-C 8 \_2$ & $1.507(4)$ \\
\hline C6_1-C7_1 & $1.383(5)$ & C6_2-C7_2 & $1.383(5)$ \\
\hline C6_1-H6_1 & 0.9500 & C6_2-H6_2 & 0.9500 \\
\hline C7_1-C7A_1 & $1.381(5)$ & C7_2-C7A_2 & $1.374(5)$ \\
\hline C10_1-C11_1 & $1.517(5)$ & C10_2-C11_2 & $1.532(5)$ \\
\hline C10_1-H10A_1 & 0.9900 & C10_2-H10C_2 & 0.9900 \\
\hline C10_1-H10B_1 & 0.9900 & C10_2-H10D_2 & 0.9900 \\
\hline $\mathrm{C} 11 \_1-\mathrm{C} 12 \_1$ & $1.515(5)$ & $\mathrm{C} 11 \_2-\mathrm{C} 12 \_2$ & $1.515(5)$ \\
\hline C11_1-H11A_1 & 0.9900 & C11_2-H11C_2 & 0.9900 \\
\hline C11_1-H11B_1 & 0.9900 & C11_2-H11D_2 & 0.9900 \\
\hline C12_1-C13_1 & $1.529(5)$ & C12_2-C13_2 & $1.529(4)$ \\
\hline C13_1-C14_1 & $1.514(5)$ & C13_2-C14_2 & $1.513(5)$ \\
\hline C13_1-H13A_1 & 0.9900 & C13_2-H13C_2 & 0.9900 \\
\hline C13_1-H13B_1 & 0.9900 & C13_2-H13D_2 & 0.9900 \\
\hline C14_1-H14A_1 & 0.9900 & C14_2-H14C_2 & 0.9900 \\
\hline C14_1-H14B_1 & 0.9900 & C14_2-H14D_2 & 0.9900 \\
\hline C15_1-C17_1 & $1.507(6)$ & C15_2-C17_2 & $1.511(5)$ \\
\hline C15_1-C16_1 & $1.517(6)$ & C15_2-C16_2 & $1.540(5)$ \\
\hline C15_1-H15_1 & 1.0000 & C15_2-H15B_2 & 1.0000 \\
\hline C16_1-H16A_1 & 0.9900 & C16_2-H16C_2 & 0.9900 \\
\hline C16_1-H16B_1 & 0.9900 & C16_2-H16D_2 & 0.9900 \\
\hline C17_1-H17A_1 & 0.9800 & C17_2-H17D_2 & 0.9800 \\
\hline C17_1-H17B_1 & 0.9800 & C17_2-H17E_2 & 0.9800 \\
\hline C17_1-H17C_1 & 0.9800 & C17_2-H17F_2 & 0.9800 \\
\hline
\end{tabular}




\begin{tabular}{|c|c|c|c|}
\hline $\mathrm{O} 1 \_1-\mathrm{S} 1 \_1-\mathrm{N} 2 \_1$ & $105.13(14)$ & $\mathrm{O} 1 \_2-\mathrm{S} 1 \_2-\mathrm{N} 2 \_2$ & $105.61(14)$ \\
\hline $\mathrm{O} 1 \_1-\mathrm{S} 1 \_1-\mathrm{C} 7 \mathrm{~A} \_1$ & $107.49(14)$ & O1_2-S1_2-C7A_2 & $107.10(14)$ \\
\hline $\mathrm{N} 2 \_1-\mathrm{S} 1 \_1-\mathrm{C} 7 \mathrm{~A} \_1$ & $87.31(14)$ & $\mathrm{N} 2 \_2-\mathrm{S} 1 \_2-\mathrm{C} 7 \mathrm{~A}-2$ & $87.17(14)$ \\
\hline $\mathrm{C} 12 \_1-\mathrm{O} 6 \_1-\mathrm{C} 15 \_1$ & $108.7(3)$ & $\mathrm{C} 12 \_2-\mathrm{O} 6 \_2-\mathrm{C} 15 \_2$ & $105.3(2)$ \\
\hline $\mathrm{C} 121-\mathrm{O} 71-\mathrm{C} 161$ & $106.6(3)$ & $\mathrm{C} 122-\mathrm{O} 72-\mathrm{C} 162$ & $106.7(2)$ \\
\hline $\mathrm{C} 3 \_1-\mathrm{N} 2 \_1-\mathrm{C} 9 \_1$ & $126.8(3)$ & $\mathrm{C} 3 \_2-\mathrm{N} 2{ }_{2}-\mathrm{C} 9 \_2$ & $125.4(3)$ \\
\hline $\mathrm{C} 3 \_1-\mathrm{N} 2 \_1-\mathrm{S} 1 \_1$ & $117.7(2)$ & $\mathrm{C} 3 \_2-\mathrm{N} 2 \_2-\mathrm{S} 1 \_2$ & $118.0(2)$ \\
\hline $\mathrm{C} 9 \_1-\mathrm{N} 2 \_1-\mathrm{S} 1 \_1$ & $112.9(2)$ & $\mathrm{C} 9 \_2-\mathrm{N} 2 \_2-\mathrm{S} 1 \_2$ & $113.8(2)$ \\
\hline $\mathrm{O} 41-\mathrm{N} 31-\mathrm{O} 31$ & $124.5(3)$ & $\mathrm{O} 42-\mathrm{N} 32-\mathrm{O} 32$ & $124.6(3)$ \\
\hline O4_1-N3_1-C7_1 & $118.5(3)$ & $\mathrm{O} 4 \_2-\mathrm{N} 3 \_2-\mathrm{C} 7 \_2$ & $118.6(3)$ \\
\hline O3_1-N3_1-C7_1 & $117.1(3)$ & $\mathrm{O} 3 \_2-\mathrm{N} 3 \_2-\mathrm{C} 7 \_2$ & $116.8(3)$ \\
\hline C9_1-N4_1-C10_1 & $117.9(3)$ & C9_2-N4_2-C10_2 & $118.8(3)$ \\
\hline C9_1-N4_1-C14_1 & $126.5(3)$ & C9_2-N4_2-C14_2 & $126.7(3)$ \\
\hline $\mathrm{C} 10 \_1-\mathrm{N} 4 \_1-\mathrm{C} 14 \_1$ & $113.5(3)$ & $\mathrm{C} 10 \_2-\mathrm{N} 4 \_2-\mathrm{C} 14 \_2$ & $113.5(3)$ \\
\hline $\mathrm{O} 2 \_1-\mathrm{C} 3 \_1-\mathrm{N} 2 \_1$ & $125.5(3)$ & $\mathrm{O} 2 \_2-\mathrm{C} 3 \_2-\mathrm{N} 2 \_2$ & $125.8(3)$ \\
\hline $\mathrm{O} 2 \_1-\mathrm{C} 3 \_1-\mathrm{C} 3 \mathrm{~A} \_1$ & $126.8(3)$ & $\mathrm{O} 2 \_2-\mathrm{C} 3 \_2-\mathrm{C} 3 \mathrm{~A} \_2$ & $126.5(3)$ \\
\hline N2_1-C3_1-C3A_1 & $107.7(3)$ & $\mathrm{N} 2 \_2-\mathrm{C} 3 \_2-\mathrm{C} 3 \mathrm{~A} \_2$ & $107.7(3)$ \\
\hline C7A_1-C3A_1-C4_1 & $121.8(3)$ & C7A_2-C3A_2-C4_2 & $121.6(3)$ \\
\hline C7A_1-C3A_1-C3_1 & $112.9(3)$ & C7A_2-C3A_2-C3_2 & $112.7(3)$ \\
\hline C4_1-C3A_1-C3_1 & $125.2(3)$ & C4 2 2-C3A_2-C3_2 & $125.7(3)$ \\
\hline C3A_1-C4_1-C5_1 & $117.8(3)$ & C3A_2-C4_2-C5_2 & $117.9(3)$ \\
\hline C3A_1-C4_1-H4_1 & 121.1 & $\mathrm{C} 3 \mathrm{~A} \_2-\mathrm{C} 4 \_2-\mathrm{H} 4 \_2$ & 121.1 \\
\hline $\mathrm{C} 5 \_1-\mathrm{C} 4 \_1-\mathrm{H} 4 \_1$ & 121.1 & $\mathrm{C} 5 \_2-\mathrm{C} 4 \_2-\mathrm{H} 4 \_2$ & 121.1 \\
\hline C6_1-C5_1-C4_1 & $120.9(3)$ & $\mathrm{C} 6 \_2-\mathrm{C} 5 \_2-\mathrm{C} 4 \_2$ & $121.2(3)$ \\
\hline C6_1-C5_1-C8_1 & $119.1(3)$ & C6 $22-\mathrm{C} 5 \_2-\mathrm{C} 8 \_2$ & $118.9(3)$ \\
\hline $\mathrm{C} 4 \_1-\mathrm{C} 5 \_1-\mathrm{C} 8 \_1$ & $120.0(3)$ & $\mathrm{C} 4 \_2-\mathrm{C} 5 \_2-\mathrm{C} 8 \_2$ & $119.8(3)$ \\
\hline C7_1-C6_1-C5_1 & $119.3(3)$ & C7_2-C6_2-C5_2 & $119.1(3)$ \\
\hline C7_1-C6_1-H6_1 & 120.3 & C7_2-C6_2-H6_2 & 120.5 \\
\hline $\mathrm{C} 5 \_1-\mathrm{C} 6 \_1-\mathrm{H} 6 \_1$ & 120.3 & C5_2-C6_2-H6_2 & 120.5 \\
\hline C7A_1-C7_1-C6_1 & $120.6(3)$ & C7A_2-C7_2-C6_2 & $120.9(3)$ \\
\hline C7A_1-C7_1-N3_1 & $120.3(3)$ & C7A_2-C7_2-N3_2 & $120.6(3)$ \\
\hline C6_1-C7_1-N3_1 & $119.1(3)$ & $\mathrm{C} 6 \_2-\mathrm{C} 7 \_2-\mathrm{N} 3 \_2$ & $118.5(3)$ \\
\hline C3A_1-C7A_1-C7_1 & $119.4(3)$ & C7_2-C7A_2-C3A_2 & $119.2(3)$ \\
\hline $\mathrm{C} 3 \mathrm{~A} \_1-\mathrm{C} 7 \mathrm{~A} \_1-\mathrm{S} 1 \_1$ & $113.6(2)$ & C7_2-C7A_2-S1_2 & $127.5(3)$ \\
\hline C7_1-C7A_1-S1_1 & $126.9(3)$ & C3A_2-C7A_2-S1_2 & $113.3(2)$ \\
\hline $\mathrm{F} 2 \_1-\mathrm{C} 8 \_1-\mathrm{F} 1 \_1$ & $109.6(3)$ & $\mathrm{F} 2 \_2-\mathrm{C} 8 \_2-\mathrm{F} 1 \_2$ & $107.2(3)$ \\
\hline $\mathrm{F} 2 \_1-\mathrm{C} 8 \_1-\mathrm{F} 3 \_1$ & $104.7(3)$ & $\mathrm{F} 2 \_2-\mathrm{C} 8 \_2-\mathrm{F} 3 \_2$ & $107.2(3)$ \\
\hline $\mathrm{F} 1 \_1-\mathrm{C} 8 \_1-\mathrm{F} 3 \_1$ & $105.5(3)$ & $\mathrm{F} 1 \_2-\mathrm{C} 8 \_2-\mathrm{F} 3 \_2$ & $106.2(3)$ \\
\hline $\mathrm{F} 2 \_1-\mathrm{C} 8 \_1-\mathrm{C} 5 \_1$ & $113.5(3)$ & $\mathrm{F} 2 \_2-\mathrm{C} 8 \_2-\mathrm{C} 5 \_2$ & $113.0(3)$ \\
\hline $\mathrm{F} 1 \_1-\mathrm{C} 8 \_1-\mathrm{C} 5 \_1$ & $112.6(3)$ & $\mathrm{F} 1 \_2-\mathrm{C} 8 \_2-\mathrm{C} 5 \_2$ & $111.2(3)$ \\
\hline F3_1-C8_1-C5 1 & $110.3(3)$ & F3 $2-\mathrm{C} 8 \_2-\mathrm{C} 5 \_2$ & $111.7(3)$ \\
\hline $\mathrm{O} 5 \_1-\mathrm{C} 9 \_1-\mathrm{N} 4 \_1$ & $125.8(3)$ & $\mathrm{O} 5 \_2-\mathrm{C} 9 \_2-\mathrm{N} 4 \_2$ & $126.3(3)$ \\
\hline $\mathrm{O} 5 \_1-\mathrm{C} 9{ }_{-}^{-} 1-\mathrm{N} 2{ }_{-}^{-} 1$ & $117.8(3)$ & $\mathrm{O} 5 \_2-\mathrm{C} 9 \_2-\mathrm{N} 2{ }^{-} 2$ & $118.6(3)$ \\
\hline N4_1-C9_1-N2_1 & $116.5(3)$ & $\mathrm{N} 4 \_2-\mathrm{C} 9 \_2-\mathrm{N} 2 \_2$ & $115.1(3)$ \\
\hline N4_1-C10_1-C11_1 & $110.5(3)$ & N4_2-C10_2-C11_2 & $109.7(3)$ \\
\hline N4_1-C10_1-H10A_1 & 109.5 & N4_2-C10_2-H10C_2 & 109.7 \\
\hline C11_1-C10_1-H10A_1 & 109.5 & C11_2-C10_2-H10C_2 & 109.7 \\
\hline N4_1-C10_1-H10B_1 & 109.5 & N4_2-C10_2-H10D_2 & 109.7 \\
\hline
\end{tabular}




\begin{tabular}{|c|c|c|c|}
\hline C11_1-C10_1-H10B_1 & 109.5 & C11_2-C10_2-H10D_2 & 109.7 \\
\hline H10A_1-C10_1-H10B_1 & 108.1 & H10C_2-C10_2-H10D_2 & 108.2 \\
\hline C12_1-C11_1-C10_1 & $110.6(3)$ & C12_2-C11_2-C10_2 & $111.0(3)$ \\
\hline C12_1-C11_1-H11A_1 & 109.5 & C12_2-C11_2-H11C_2 & 109.4 \\
\hline C10_1-C11_1-H11A_1 & 109.5 & C10_2-C11_2-H11C_2 & 109.4 \\
\hline C12_1-C11_1-H11B_1 & 109.5 & C12_2-C11_2-H11D_2 & 109.4 \\
\hline C10_1-C11_1-H11B_1 & 109.5 & C10_2-C11_2-H11D_2 & 109.4 \\
\hline H11A_1-C11_1-H11B_1 & 108.1 & H11C_2-C11_2-H11D_2 & 108.0 \\
\hline O7_1-C12_1-O6_1 & $106.8(3)$ & O7_2-C12_2-O6_2 & $105.6(3)$ \\
\hline O7_1-C12_1-C11_1 & $109.5(3)$ & O7_2-C12_2-C11_2 & $110.0(3)$ \\
\hline O6_1-C12_1-C11_1 & $109.9(3)$ & O6_2-C12_2-C11_2 & $108.5(3)$ \\
\hline O7_1-C12_1-C13_1 & $111.6(3)$ & O7_2-C12_2-C13_2 & $111.5(3)$ \\
\hline O6_1-C12_1-C13_1 & $108.1(3)$ & O6_2-C12_2-C13_2 & $111.0(3)$ \\
\hline $\mathrm{C} 1 \overline{1} \_1-\mathrm{C} 1 \overline{2} \_1-\mathrm{C} 1 \overline{3} \_1$ & $110.8(3)$ & $\mathrm{C} 1 \overline{1} \_2-\mathrm{C} 1 \overline{2} \_2-\mathrm{C} 1 \overline{3} \_2$ & $110.1(3)$ \\
\hline C14_1-C13_1-C12_1 & $109.6(3)$ & C14_2-C13_2-C12_2 & $109.8(3)$ \\
\hline C14_1-C13_1-H13A_1 & 109.8 & C14_2-C13_2-H13C_2 & 109.7 \\
\hline C12_1-C13_1-H13A_1 & 109.8 & C12_2-C13_2-H13C_2 & 109.7 \\
\hline C14_1-C13_1-H13B_1 & 109.8 & C14_2-C13_2-H13D_2 & 109.7 \\
\hline C12_1-C13_1-H13B_1 & 109.8 & C12_2-C13_2-H13D_2 & 109.7 \\
\hline H13A_1-C13_1-H13B_1 & 108.2 & H13C_2-C13_2-H13D_2 & 108.2 \\
\hline N4_1-C14_1-C13_1 & $110.8(3)$ & N4_2-C14_2-C13_2 & $110.7(3)$ \\
\hline N4_1-C14_1-H14A_1 & 109.5 & N4_2-C14_2-H14C_2 & 109.5 \\
\hline C13_1-C14_1-H14A_1 & 109.5 & C13_2-C14_2-H14C_2 & 109.5 \\
\hline N4_1-C14_1-H14B_1 & 109.5 & N4_2-C14_2-H14D_2 & 109.5 \\
\hline C13_1-C14_1-H14B_1 & 109.5 & C13_2-C14_2-H14D_2 & 109.5 \\
\hline H14A_1-C14_1-H14B_1 & 108.1 & H14C_2-C14_2-H14D_2 & 108.1 \\
\hline O6_1-C15_1-C17_1 & $109.7(3)$ & O6_2-C15_2-C17_2 & $108.8(3)$ \\
\hline O6_1-C15_1-C16_1 & $101.4(3)$ & O6_2-C15_2-C16_2 & $103.1(3)$ \\
\hline C17_1-C15_1-C16_1 & $115.3(3)$ & C17_2-C15_2-C16_2 & $115.1(3)$ \\
\hline O6_1-C15_1-H15_1 & 110.0 & O6_2-C15_2-H15B_2 & 109.9 \\
\hline C17_1-C15_1-H15_1 & 110.0 & C17_2-C15_2-H15B_2 & 109.9 \\
\hline C16_1-C15_1-H15_1 & 110.0 & C16_2-C15_2-H15B_2 & 109.9 \\
\hline O7_1-C16_1-C15_1 & $103.3(3)$ & O7_2-C16_2-C15_2 & $105.4(3)$ \\
\hline O7_1-C16_1-H16A_1 & 111.1 & O7_2-C16_2-H16C_2 & 110.7 \\
\hline C15_1-C16_1-H16A_1 & 111.1 & C15_2-C16_2-H16C_2 & 110.7 \\
\hline O7_1-C16_1-H16B_1 & 111.1 & O7_2-C16_2-H16D_2 & 110.7 \\
\hline C15_1-C16_1-H16B_1 & 111.1 & C15_2-C16_2-H16D_2 & 110.7 \\
\hline H16A_1-C16_1-H16B_1 & 109.1 & H16C_2-C16_2-H16D_2 & 108.8 \\
\hline C15_1-C17_1-H17A_1 & 109.5 & C15_2-C17_2-H17D_2 & 109.5 \\
\hline C15_1-C17_1-H17B_1 & 109.5 & C15_2-C17_2-H17E_2 & 109.5 \\
\hline H17A_1-C17_1-H17B_1 & 109.5 & H17D_2-C17_2-H17E_2 & 109.5 \\
\hline C15_1-C17_1-H17C_1 & 109.5 & C15_2-C17_2-H17F_2 & 109.5 \\
\hline H17A_1-C17_1-H17C_1 & 109.5 & H17D_2-C17_2-H17F_2 & 109.5 \\
\hline H17B_1-C17_1-H17C_1 & 109.5 & H17E_2-C17_2-H17F_2 & 109.5 \\
\hline $\mathrm{O} 1 \_1-\mathrm{S} 1 \_1-\mathrm{N} 2 \_1-\mathrm{C} 3 \_1$ & $113.0(2)$ & $\mathrm{O} 1 \_2-\mathrm{S} 1 \_2-\mathrm{N} 2 \_2-\mathrm{C} 3 \_2$ & $-114.6(2)$ \\
\hline C7A_1-S1_1-N2_1-C3_1 & $5.6(3)$ & C7A_2-S1_2-N2_2-C3_2 & $-7.6(2)$ \\
\hline $\mathrm{O} 1 \_1-\mathrm{S} 1 \_1-\mathrm{N} 2 \_1-\mathrm{C} 9 \_1$ & $-84.0(2)$ & $\mathrm{O} 1 \_2-\mathrm{S} 1 \_2-\mathrm{N} 2 \_2-\mathrm{C} 9 \_2$ & $83.3(2)$ \\
\hline
\end{tabular}




\begin{tabular}{|c|c|c|c|}
\hline $\mathrm{C} 7 \mathrm{~A} \_1-\mathrm{S} 1 \_1-\mathrm{N} 2 \_1-\mathrm{C} 9 \_1$ & $168.6(2)$ & $\mathrm{C} 7 \mathrm{~A} \_2-\mathrm{S} 1 \_2-\mathrm{N} 2 \_2-\mathrm{C} 9 \_2$ & $-169.7(2)$ \\
\hline $\mathrm{C} 9 \_1-\mathrm{N} 2 \_1-\mathrm{C} 3 \_1-\mathrm{O} 2 \_1$ & $8.8(6)$ & $\mathrm{C} 9 \_2-\mathrm{N} 2 \_2-\mathrm{C} 3 \_2-\mathrm{O} 2 \_2$ & $-6.6(5)$ \\
\hline $\mathrm{S} 1 \_1-\mathrm{N} 2 \_1-\mathrm{C} 3 \_1-\mathrm{O} 2 \_1$ & $169.0(3)$ & $\mathrm{S} 1 \_2-\mathrm{N} 2 \_2-\mathrm{C} 3 \_2-\mathrm{O} 2 \_2$ & $-166.4(3)$ \\
\hline C9 $1-\mathrm{N} 2 \_1-\mathrm{C} 3 \_1-\mathrm{C} 3 \mathrm{~A} \_1$ & $-169.5(3)$ & C9_2-N2_2-C3_2-C3A_2 & $171.4(3)$ \\
\hline $\mathrm{S} 1 \_1-\mathrm{N} 2 \_1-\mathrm{C} 3 \_1-\mathrm{C} 3 \mathrm{~A} \_1$ & $-9.2(3)$ & $\mathrm{S} 1 \_2-\mathrm{N} 2 \_2-\mathrm{C} 3 \_2-\mathrm{C} 3 \mathrm{~A} \_2$ & $11.6(3)$ \\
\hline O2_1-C3_1-C3A_1-C7A_1 & $-169.4(3)$ & O2_2-C3_2-C3A_2-C7A_2 & $167.7(3)$ \\
\hline N2_1-C3_1-C3A_1-C7A_1 & $8.9(4)$ & N2_2-C3_2-C3A_2-C7A_2 & $-10.3(4)$ \\
\hline $\mathrm{O} 2 \_1-\mathrm{C} 3 \_1-\mathrm{C} 3 \mathrm{~A} \_1-\mathrm{C} 4 \_1$ & $8.9(6)$ & $\mathrm{O} 2 \_2-\mathrm{C} 3 \_2-\mathrm{C} 3 \mathrm{~A} \_2-\mathrm{C} 4 \_2$ & $-11.0(5)$ \\
\hline $\mathrm{N} 2 \_1-\mathrm{C} 3 \_1-\mathrm{C} 3 \mathrm{~A} \_1-\mathrm{C} 4 \_1$ & $-172.9(3)$ & $\mathrm{N} 2 \_2-\mathrm{C} 3 \_2-\mathrm{C} 3 \mathrm{~A} \_2-\mathrm{C} 4 \_2$ & $170.9(3)$ \\
\hline C7A_1-C3A_1-C4_1-C5_1 & $2.3(5)$ & C7A_2-C3A_2-C4_2-C5_2 & $-2.5(5)$ \\
\hline C3_1-C3A_1-C4_1-C5_1 & $-175.8(3)$ & C3_2-C3A_2-C4_2-C5_2 & $176.1(3)$ \\
\hline C3A_1-C4_1-C5_1-C6_1 & $-0.4(5)$ & C3A_2-C4_2-C5_2-C6_2 & $1.1(5)$ \\
\hline C3A_1-C4_1-C5_1-C8_1 & $178.0(3)$ & C3A_2-C4_2-C5_2-C8_2 & $-176.4(3)$ \\
\hline C4_1-C5_1-C6_1-C7_1 & $-1.0(5)$ & C4_2-C5_2-C6_2-C7_2 & $0.1(5)$ \\
\hline C8_1-C5_1-C6_1-C7_1 & $-179.3(3)$ & C8_2-C5_2-C6_2-C7_2 & $177.6(3)$ \\
\hline C5_1-C6_1-C7_1-C7A_1 & $0.5(5)$ & C5_2-C6_2-C7_2-C7A_2 & $0.1(4)$ \\
\hline $\mathrm{C} 5 \_1-\mathrm{C} 6 \_1-\mathrm{C} 7 \_1-\mathrm{N} 3 \_1$ & $-179.6(3)$ & $\mathrm{C} 5 \_2-\mathrm{C} 6 \_2-\mathrm{C} 7 \_2-\mathrm{N} 3 \_2$ & $-179.8(3)$ \\
\hline O4_1-N3_1-C7_1-C7A_1 & $-173.3(3)$ & O4_2-N3_2-C7_2-C7A_2 & $169.6(3)$ \\
\hline O3_1-N3_1-C7_1-C7A_1 & $7.5(5)$ & O3_2-N3_2-C7_2-C7A_2 & $-11.4(4)$ \\
\hline O4_1-N3_1-C7_1-C6_1 & $6.8(4)$ & $\mathrm{O} 4 \_2-\mathrm{N} 3 \_2-\mathrm{C} 7 \_2-\mathrm{C} 6 \_2$ & $-10.5(4)$ \\
\hline O3_1-N3_1-C7_1-C6_1 & $-172.5(3)$ & O3_2-N3_2-C7_2-C6_2 & $168.5(3)$ \\
\hline C4_1-C3A_1-C7A_1-C7_1 & $-2.8(5)$ & C6_2-C7_2-C7A_2-C3A_2 & $-1.5(4)$ \\
\hline C3_1-C3A_1-C7A_1-C7_1 & $175.5(3)$ & N3_2-C7_2-C7A_2-C3A_2 & $178.4(3)$ \\
\hline C4_1-C3A_1-C7A_1-S1_1 & $176.5(2)$ & C6_2-C7_2-C7A_2-S1_2 & $176.9(2)$ \\
\hline C3_1-C3A_1-C7A_1-S1_1 & $-5.2(3)$ & N3_2-C7_2-C7A_2-S1_2 & $-3.2(4)$ \\
\hline C6_1-C7_1-C7A_1-C3A_1 & $1.4(4)$ & C4_2-C3A_2-C7A_2-C7_2 & $2.7(5)$ \\
\hline N3_1-C7_1-C7A_1-C3A_1 & $-178.6(3)$ & C3_2-C3A_2-C7A_2-C7_2 & $-176.1(3)$ \\
\hline C6_1-C7_1-C7A_1-S1_1 & $-177.8(2)$ & $\mathrm{C} 4 \_2-\mathrm{C} 3 \mathrm{~A} \_2-\mathrm{C} 7 \mathrm{~A} \_2-\mathrm{S} 1 \_2$ & $-175.9(2)$ \\
\hline N3_1-C7_1-C7A_1-S1_1 & $2.2(4)$ & C3_2-C3A_2-C7A_2-S1_2 & $5.3(3)$ \\
\hline O1_1-S1_1-C7A_1-C3A_1 & $-104.9(2)$ & O1_2-S1_2-C7A_2-C7_2 & $-72.1(3)$ \\
\hline N2_1-S1_1-C7A_1-C3A_1 & $0.1(2)$ & N2_2-S1_2-C7A_2-C7_2 & $-177.6(3)$ \\
\hline O1_1-S1_1-C7A_1-C7_1 & $74.3(3)$ & O1_2-S1_2-C7A_2-C3A_2 & $106.3(2)$ \\
\hline $\mathrm{N} 2 \_1-\mathrm{S} 1$ 1-1-C7A_1-C7_1 & $179.3(3)$ & $\mathrm{N} 2 \_2-\mathrm{S} 1 \_2-\mathrm{C} 7 \mathrm{~A}$-2-C3A_2 & $0.9(2)$ \\
\hline C6_1-C5_1-C8_1-F2_1 & $-157.6(3)$ & C6_2-C5_2-C8_2-F2 2 2 & $157.3(3)$ \\
\hline C4_1-C5_1-C8_1-F2_1 & $24.0(5)$ & C4_2-C5_2-C8_2-F2_2 & $-25.1(4)$ \\
\hline C6_1-C5_1-C8_1-F1_1 & $77.1(4)$ & $\mathrm{C} 6 \_2-\mathrm{C} 5 \_2-\mathrm{C} 8 \_2-\mathrm{F} 1 \_2$ & $-82.1(4)$ \\
\hline $\mathrm{C} 4 \_1-\mathrm{C} 5 \_1-\mathrm{C} 8 \_1-\mathrm{F} 1 \_1$ & $-101.3(4)$ & $\mathrm{C} 4 \_2-\mathrm{C} 5 \_2-\mathrm{C} 8 \_2-\mathrm{F} 1 \_2$ & $95.5(4)$ \\
\hline C6_1-C5_1-C8_1-F3_1 & $-40.4(4)$ & C6_2-C5_2-C8_2-F3_2 & $36.4(4)$ \\
\hline C4_1-C5_1-C8_1-F3_1 & $141.2(3)$ & C4_2-C5_2-C8_2-F3_2 & $-146.1(3)$ \\
\hline $\mathrm{C} 10 \_1-\mathrm{N} 4 \_1-\mathrm{C} 9 \_1-\mathrm{O} 5 \_1$ & $-2.5(5)$ & $\mathrm{C} 10 \_2-\mathrm{N} 4 \_2-\mathrm{C} 9 \_2-\mathrm{O} 5 \_2$ & $5.0(5)$ \\
\hline C14_1-N4_1-C9_1-O5_1 & $159.9(3)$ & $\mathrm{C} 14 \_2-\mathrm{N} 4 \_2-\mathrm{C} 9 \_2-\mathrm{O} 5 \_2$ & $-162.5(3)$ \\
\hline $\mathrm{C} 10 \_1-\mathrm{N} 4 \_1-\mathrm{C} 9 \_1-\mathrm{N} 2 \_1$ & $179.8(3)$ & $\mathrm{C} 10 \_2-\mathrm{N} 4 \_2-\mathrm{C} 9 \_2-\mathrm{N} 2 \_2$ & $-176.4(3)$ \\
\hline C14_1-N4_1-C9_1-N2_1 & $-17.8(5)$ & $\mathrm{C} 14 \_2-\mathrm{N} 4 \_2-\mathrm{C} 9 \_2-\mathrm{N} 2 \_2$ & $16.1(4)$ \\
\hline $\mathrm{C} 3 \_1-\mathrm{N} 2 \_1-\mathrm{C} 9 \_1-\mathrm{O} 5 \_1$ & $127.2(4)$ & $\mathrm{C} 3 \_2-\mathrm{N} 2 \_2-\mathrm{C} 9 \_2-\mathrm{O} 5 \_2$ & $-125.1(3)$ \\
\hline $\mathrm{S} 1 \_1-\mathrm{N} 2 \_1-\mathrm{C} 9 \_1-\mathrm{O} 5 \_1$ & $-33.9(4)$ & $\mathrm{S} 1 \_2-\mathrm{N} 2 \_2-\mathrm{C} 9 \_2-\mathrm{O} 5 \_2$ & $35.4(4)$ \\
\hline $\mathrm{C} 3 \_1-\mathrm{N} 2 \_1-\mathrm{C} 9 \_1-\mathrm{N} 4 \_1$ & $-54.9(4)$ & $\mathrm{C} 3 \_2-\mathrm{N} 2 \_2-\mathrm{C} 9 \_2-\mathrm{N} 4 \_2$ & $56.2(4)$ \\
\hline $\mathrm{S} 1 \_1-\mathrm{N} 2 \_1-\mathrm{C} 9 \_1-\mathrm{N} 4 \_1$ & $144.0(2)$ & $\mathrm{S} 1 \_2-\mathrm{N} 2 \_2-\mathrm{C} 9 \_2-\mathrm{N} 4 \_2$ & $-143.3(2)$ \\
\hline C9_1-N4_1-C10_1-C11_1 & $-138.9(3)$ & C9_2-N4_2-C10_2-C11_2 & $134.3(3)$ \\
\hline
\end{tabular}




\begin{tabular}{|c|c|}
\hline $11 \_1$ & \\
\hline $\mathrm{N} 4 \_1-\mathrm{C} 10 \_1-\mathrm{C} 11 \_1-\mathrm{C} 12 \_1$ & $-55.0(4)$ \\
\hline $\mathrm{C} 16 \_1-07 \_1-\mathrm{C} 12 \_1-\mathrm{O} 6 \_1$ & $19.5(4)$ \\
\hline $\mathrm{C} 16 \_1-\mathrm{O} 7 \_1-\mathrm{C} 12 \_1-\mathrm{C} 11 \_1$ & $138.5(3)$ \\
\hline $\mathrm{C} 161-\mathrm{O} 71-\mathrm{C} 121-\mathrm{C} 131$ & $-98.5(3)$ \\
\hline $\mathrm{C} 15 \_1-\mathrm{O} 6 \_1-\mathrm{C} 12-1-\mathrm{O} 7 \_1$ & $4.1(4)$ \\
\hline $\mathrm{C} 15 \_1-\mathrm{O} 6 \_1-\mathrm{C} 12 \_1-\mathrm{C} 11 \_1$ & $-114.7(3)$ \\
\hline $\mathrm{C} 15 \_1-\mathrm{O} 6 \_1-\mathrm{C} 12 \_1-\mathrm{C} 13 \_1$ & $124.3(3)$ \\
\hline $\mathrm{C} 101-\mathrm{C} 111-\mathrm{C} 121-\mathrm{O} 71$ & $179.5(3)$ \\
\hline $\mathrm{C} 10 \_1-\mathrm{C} 11 \_1-\mathrm{C} 12 \_1-\mathrm{O} 6 \_1$ & $-63.5(4)$ \\
\hline $\mathrm{C} 10-1-\mathrm{C} 11-1-\mathrm{C} 12_{-}^{-} 1-\mathrm{C} 13 \overline{-} 1$ & $56.0(4)$ \\
\hline O7_1-C12_1-C13_1-C14_1 & $-178.6(3)$ \\
\hline $\mathrm{O} 61-\mathrm{C} 121-\mathrm{C} 13 \mathrm{1}-\mathrm{C} 141$ & $64.2(4)$ \\
\hline $\mathrm{C} 1 \overline{1} \_1-\mathrm{C} 1 \overline{2} \_1-\mathrm{C} 1 \overline{3} \_1-\mathrm{C} 1 \overline{4} \_1$ & $-56.3(4)$ \\
\hline $\mathrm{C} 9 \_1-\mathrm{N} 4 \_1-\mathrm{C} 14 \_1-\mathrm{C} 13 \_1$ & $139.5(3)$ \\
\hline $\mathrm{C} 10 \_1-\mathrm{N} 4 \_1-\mathrm{C} 14 \_1-\mathrm{C} 13 \_1$ & $-57.5(4)$ \\
\hline $\mathrm{C} 12-\mathrm{C} 13 \quad 1-\mathrm{C} 141-\mathrm{N} 41$ & $56.0(4)$ \\
\hline $\mathrm{C} 12 \_1-\mathrm{O} 6 \_1-\mathrm{C} 15 \_1-\mathrm{C} 17 \_1$ & $-146.3(3)$ \\
\hline $\mathrm{C} 12 \_1-\mathrm{O} 6 \_1-\mathrm{C} 15 \_1-\mathrm{C} 16 \_1$ & $-23.9(4)$ \\
\hline $\mathrm{C} 12 \_1-\mathrm{O} 7 \_1-\mathrm{C} 16 \_1-\mathrm{C} 15 \_1$ & $-34.1(4)$ \\
\hline $\mathrm{O} 61-\mathrm{C} 151-\mathrm{C} 161-\mathrm{O} 71$ & $35.2(4)$ \\
\hline $\mathrm{C} 1 \overline{7} 1-\mathrm{C} 1 \overline{5} 1-\mathrm{C} 1 \overline{6} 1-\mathrm{O} \overline{7} 1$ & $153.6(3)$ \\
\hline
\end{tabular}

C14_2-N4_2-C10_2-C11_2 -56.6(4)

N4_2-C10_2-C11_2-C12_2 55.3 (4)

C16_2-O7_2-C12_2-O6_2 -32.4 (3)

C16_2-O7_2-C12_2-C11_2 -149.2 (3)

C16_2-O7_2-C12_2-C13_2 88.3 (3)

C15_2-O6_2-C12_2-O7_2 38.7 (3)

C15_2-O6_2-C12_2-C11_2 156.5 (3)

$\mathrm{C} 15 \_2-\mathrm{O} 6 \_2-\mathrm{C} 12 \_2-\mathrm{C} 13 \_2-82.3$ (3)

C10_2-C11_2-C12_2-O7_2 -179.5 (3)

C10_2-C11_2-C12_2-O6_2 65.5 (3)

$\mathrm{C} 10 \_2-\mathrm{C} 11 \_2-\mathrm{C} 12 \_2-\mathrm{C} 13 \_2-56.1$ (4)

O7_2-C12_2-C13_2-C14_ 2 178.9(3)

O6_2-C12_2-C13_2-C14_2 -63.6(4)

C11_2-C12_2-C13_2-C14_2 56.5 (3)

C9_2-N4_2-C14_2-C13_2 -133.8(3)

$\mathrm{C} 10 \_2-\mathrm{N} 4 \_2-\mathrm{C} 14 \_2-\mathrm{C} 13 \_2 \quad 58.2$ (4)

$\mathrm{C} 12 \_2-\mathrm{C} 13 \_2-\mathrm{C} 14 \_2-\mathrm{N} 4 \_2-56.7$ (3)

C12_2-O6_2-C15_2-C17_2 -151.2(3)

C12_2-O6_2-C15_2-C16_2 -28.6 (3)

$\mathrm{C} 12 \_2-\mathrm{O} 7 \_2-\mathrm{C} 16 \_2-\mathrm{C} 15 \_2 \quad 13.8(3)$

O6_2-C15_2-C16_2-O7_ 9.2 (3)

$\mathrm{C} 1 \overline{7} \_2-\mathrm{C} 1 \overline{5} \_2-\mathrm{C} 1 \overline{6} \_2-\mathrm{O} 7 \_227.5$ (3) 